\title{
MULTIPLE CRITERIA DECISION ANALYSIS USING PRIORITISED INTERVAL TYPE-2 FUZZY AGGREGATION OPERATORS AND ITS APPLICATION TO SITE SELECTION
}

\author{
Ting-Yu CHEN \\ Department of Industrial and Business Management, Graduate Institute of Business and Management, \\ College of Management, Chang Gung University, Division of Cerebrovascular Disease, \\ Department of Neurology, Linkou Chang Gung Memorial Hospital, 259, \\ Wenhua 1st Road, Guishan,Taoyuan 333, Taiwan
}

Received 01 February 2013; accepted 02 November 2013

\begin{abstract}
The theory of interval type-2 fuzzy sets provides an intuitive and computationally feasible method of addressing uncertain and ambiguous information in decision-making fields. This paper aims to develop a prioritised interval type-2 fuzzy aggregation operator and apply it to multiple criteria decision analysis with prioritised criteria. This paper considers situations in which a relationship between the criteria exists such that a lack of satisfaction by the higher priority criteria cannot be readily compensated by the satisfaction of lower priority criteria. This paper introduces the developed prioritised interval type-2 fuzzy aggregation operator to address the problem of criteria aggregation in this environment. To demonstrate the feasibility of the proposed operator, this paper provides a multiple criteria decision-making method that uses the prioritised interval type- 2 fuzzy aggregation operator, and the method is illustrated with a practical application to landfill site selection.
\end{abstract}

Keywords: interval type-2 fuzzy set, prioritised interval type-2 fuzzy aggregation operator, multiple criteria decision analysis, landfill site selection.

JEL Classification: C44, D81, R53.

\section{Introduction}

Uncertain and imprecise information are commonly present in practical multiple criteria decision analysis (MCDA) situations (Han, Liu 2011) because decision-makers might not easily express their subjective assessments using exact and crisp values. Modelling the uncertainty in human subjective management becomes increasingly important when addressing MCDA problems (Zavadskas, Turskis 2011; Liou, Tzeng 2012). Regardless of the settings in complex or linguistic decision environments, interval type-2 fuzzy (IT2F) sets

Corresponding author Ting-Yu Chen

E-mail: tychen@mail.cgu.edu.tw 
offer a useful means for managing the uncertainty and imprecision that arise from mental phenomena. IT2F sets are the most widely used type of type-2 fuzzy sets (Zadeh 1975) because of their relative simplicity (Mendel 2007). IT2F sets are superior to ordinary fuzzy sets because they are able to model second-order uncertainties (Greenfield et al. 2009). The membership values of IT2F sets take the form of crisp intervals, and thus, the computations associated with IT2F sets are manageable (Mendel et al. 2006). Currently, the IT2F theory has been successfully applied in practical MCDA or assessment problems (Zhai, Mendel 2011; Lai, Chen 2015; Gilan et al. 2012; Stanujkic et al. 2012; Wang et al. 2012; Wei et al. 2012).

Most of the existing MCDA methods in the IT2F environment assume that all of the criteria are at the same priority level. Yager $(2008,2009)$ indicated that the relationship between safety and cost is typical of the problem of prioritised criteria. For example, when parents select a bicycle for their children based on the criteria of safety and cost, they must not allow a benefit with respect to the cost to compensate for a loss in safety. Importantly, safety has a higher priority than cost. Decision-makers prefer to consider the satisfaction of higher-priority criteria, such as the safety criterion mentioned above; thus, it is no longer appropriate to employ the existing MCDA methods with IT2F sets. Yager (2004, 2008, 2009) and Yager et al. (2011) introduced the concept of prioritised aggregation operators to address an aggregation problem in which a prioritisation relationship exists among the criteria. Yager (2008) presented prioritised aggregation operators by modelling the prioritisation of criteria using weights associated with the criteria that are dependent on the satisfaction of higher-priority criteria. Yager (2009) used importance weights to enforce this prioritisation imperative and applied his proposed priority-based importance weights to a case in which the scope of the criteria aggregation was an ordered weighted averaging (OWA) type of aggregation. Yager (2009) and Yager et al. (2011) studied prioritised "and" and "or" operators and employed these in aggregation problems that exhibited prioritisation relationships among the aggregated arguments.

Prioritised aggregation operators have been extended to the fuzzy decision environment. For example, Zhao et al. (2013) proposed selected prioritised aggregation operators for aggregating triangular fuzzy information and subsequently developed certain models for solving triangular fuzzy multiple criteria group decision-making problems in which the criteria and the experts display different priority levels. Xu et al. (2011) developed an intuitionistic fuzzy prioritised OWA operator to provide a novel method for solving prioritised MCDA problems in the intuitionistic fuzzy environment. Yu (2012) proposed a generalised intuitionistic fuzzy prioritised weighted geometric operator based on an Archimedean $\mathrm{t}$-conorm and t-norm and developed a multiple criteria group decision-making method using this operator. Yu et al. (2012) proposed certain prioritised aggregation operators in the context of interval-valued intuitionistic fuzzy sets, i.e., the prioritised weighted average operator and the prioritised weighted geometric operator, and applied them to group decision-making. Motivated by the idea of Yager's prioritised "and" and "or" operators, $\mathrm{Li}$ and $\mathrm{He}$ (2012) developed intuitionistic fuzzy prioritised "and" and "or" operators and used them to aggregate intuitionistic fuzzy information when the criteria existed in different priority levels. Yu and $\mathrm{Xu}$ (2013) extended the prioritised aggregation operators developed 
by Yager (2008) to introduce the concepts of the prioritised intuitionistic fuzzy aggregation operator. Furthermore, $\mathrm{Yu}$ and $\mathrm{Xu}$ (2013) proposed a prioritised intuitionistic fuzzy OWA operator using an intuitionistic fuzzy basic unit monotonic function. In the context of hesitant fuzzy sets, Wei (2012) developed certain hesitant fuzzy prioritised aggregation operators and applied them to address MCDA problems in which the criteria existed in different priority levels.

Prioritised aggregation operators have been used in the environments of triangular fuzzy numbers (Zhao et al. 2013), intuitionistic fuzzy sets (Xu et al. 2011; Yu 2012; Li, He 2012; Yu, Xu 2013), interval-valued intuitionistic fuzzy sets (Yu et al. 2012), and hesitant fuzzy sets (Wei 2012). However, the existing studies on fuzzy prioritised aggregation operators were not based on an environment of IT2F sets. Due to a lack of data, time pressure, or the limited attention of decision-makers and information-processing capabilities, the decision-makers often make their decisions on real-world problems in linguistic or subjective environments (Chen 2012; Rajpathak et al. 2012). Therefore, IT2F sets are useful for conveniently modelling impressions and quantifying the ambiguous nature of linguistic judgments (Chen, Lee 2010; Zhang, Z., Zhang, S. 2013). IT2F sets have been developed and applied to MCDA; nevertheless, there have been few attempts to investigate IT2F MCDA problems with prioritised criteria. Considering the usefulness of IT2F sets in decision-making, this study is devoted to constructing a new MCDA method with a developed prioritised IT2F aggregation operator. This method is completely different from the existing prioritised aggregation operators in the fuzzy environment. Based on the IT2F framework, this paper employs the popular fuzzy numbers with trapezoidal forms (as employed by Chen (2011, 2012), Baležentis and Zeng (2013), Chen et al. (2013), and Zhang, Z., Zhang, S. (2013)), referred to in this work as interval type-2 trapezoidal fuzzy (IT2 TrF) numbers, to develop a new concept of prioritised IT2F aggregation operators and to establish IT2F MCDA procedures using IT2F arithmetic operations and the concept of signed distances on IT2TrF numbers.

In the environment of IT2F sets, this paper aims to develop a novel prioritised IT2F aggregation operator and to propose an MCDA method that can address multiple criteria decision-making problems in which a prioritisation relationship exists among the evaluative criteria. Based on IT2TrF numbers (Chen 2011, 2012; Chen et al. 2013), a procedure is presented for determining priority-based weights, and several valuable and important properties are investigated. Next, this paper proposes a new concept of prioritised IT2F aggregation operators and presents a useful approach via the developed operators to aggregate the IT2TrF ratings of decision alternatives with respect to prioritised criteria. The concept of signed distances is used to compare synthetic evaluations of the alternatives, and an algorithmic procedure for multiple criteria decision analysis is presented using prioritised IT2F aggregation operators. The feasibility and applicability of the proposed method are illustrated in a practical problem of landfill site selection.

Incinerators and landfills are public facilities used for garbage disposal purposes. Although most waste can be handled via incineration, the ashes generated by an incinerator must be completely disposed of in a landfill. However, landfill capacity is limited. The most economical approach to solving the problem of insufficient landfill capacity is to search for 
a new landfill location and to establish landfill facilities to treat continuously produced solid wastes. The criteria that must be considered when selecting a landfill site are complicated or ambiguous, and prioritisation of the relationships among these criteria may exist based on input from the governing authority. Therefore, this paper uses IT2F sets to capture the imprecise or uncertain practical information that is often observed with landfill decision-making problems and employs a prioritised IT2F aggregation operator to match the prioritisation relationship among the criteria.

This paper is organised as follows. Section 1 briefly reviews the concept of IT2F sets. Section 2 formulates an MCDA problem in the IT2TrF environment and develops a prioritised IT2F aggregation operator to address the MCDA problems. Section 3 illustrates the feasibility and applicability of the proposed method by applying it to the problem of landfill site selection. The last Section presents the conclusions.

\section{Basic concepts and operations of IT2F sets}

The concept of IT2F sets is used extensively throughout this paper, and thus, several relevant definitions and operations of IT2F sets are briefly reviewed in this section.

Definition 1. Let $X$ be an ordinary finite nonempty set. Let Int $([0,1])$ denote the set of all closed subintervals of $[0,1]$. The mapping $A: X \rightarrow \operatorname{Int}([0,1])$ is known as an IT2F set on $X$.

Definition 2. Let $A$ be an IT2F set on $X$. Let two ordinary fuzzy sets $A^{L}: X \rightarrow[0,1]$ and $A^{U}: X \rightarrow[0,1]$ be the lower and upper fuzzy sets, respectively, with respect to $A$. Therefore, $A(x)=\left[A^{L}(x), A^{U}(x)\right]$, where $x \in X$ and $0 \leq A^{L}(x) \leq A^{U}(x) \leq 1$. If $A(x)$ is convex and defined on a closed and bounded interval, then $A$ is known as an IT2F number on $X$.

Definition 3. Let $A^{L}\left(=\left(a_{1}^{L}, a_{2}^{L}, a_{3}^{L}, a_{4}^{L} ; h_{A}^{L}\right)\right)$ and $A^{U}\left(=\left(a_{1}^{U}, a_{2}^{U}, a_{3}^{U}, a_{4}^{U} ; h_{A}^{U}\right)\right)$ be the lower and upper trapezoidal fuzzy numbers defined on the universe of discourse $X$, where $a_{1}^{L} \leq a_{2}^{L} \leq a_{3}^{L} \leq a_{4}^{L}, a_{1}^{U} \leq a_{2}^{U} \leq a_{3}^{U} \leq a_{4}^{U}, 0 \leq h_{A}^{L} \leq h_{A}^{U} \leq 1, a_{1}^{U} \leq a_{1}^{L}, a_{4}^{L} \leq a_{4}^{U}$, and $A^{L} \subseteq A^{U}$. Let $\varsigma \in\{L, U\}$. The membership function of $A^{\varsigma}$ for each $\varsigma$ is as follows:

$$
A^{\varsigma}(x)= \begin{cases}h_{A}^{\varsigma}\left(x-a_{1}^{\varsigma}\right) /\left(a_{2}^{\varsigma}-a_{1}^{\varsigma}\right) & \text { if } a_{1}^{\varsigma} \leq x \leq a_{2}^{\varsigma}, \\ h_{A}^{\varsigma} & \text { if } a_{2}^{\varsigma} \leq x \leq a_{3}^{\varsigma}, \\ h_{A}^{\varsigma}\left(a_{4}^{\varsigma}-x\right) /\left(a_{4}^{\varsigma}-a_{3}^{\varsigma}\right) & \text { if } a_{3}^{\varsigma} \leq x \leq a_{4}^{\varsigma}, \\ 0 & \text { otherwise. }\end{cases}
$$

An IT2TrF number $A$ on $X$ (see Fig. 1) is represented by the following:

$$
A=\left[A^{L}, A^{U}\right]=\left[\left(a_{1}^{L}, a_{2}^{L}, a_{3}^{L}, a_{4}^{L} ; h_{A}^{L}\right),\left(a_{1}^{U}, a_{2}^{U}, a_{3}^{U}, a_{4}^{U} ; h_{A}^{U}\right)\right] .
$$

Definition 4. Let $A$ and $B$ be two non-negative IT2TrF numbers, and $A=\left[\left(a_{1}^{L}, a_{2}^{L}, a_{3}^{L}\right.\right.$, $\left.\left.a_{4}^{L} ; h_{A}^{L}\right),\left(a_{1}^{U}, a_{2}^{U}, a_{3}^{U}, a_{4}^{U} ; h_{A}^{U}\right)\right]$ and $B=\left[\left(b_{1}^{L}, b_{2}^{L}, b_{3}^{L}, b_{4}^{L} ; h_{B}^{L}\right),\left(b_{1}^{U}, b_{2}^{U}, b_{3}^{U}, b_{4}^{U} ; h_{B}^{U}\right)\right]$ on $X$. The ba- 


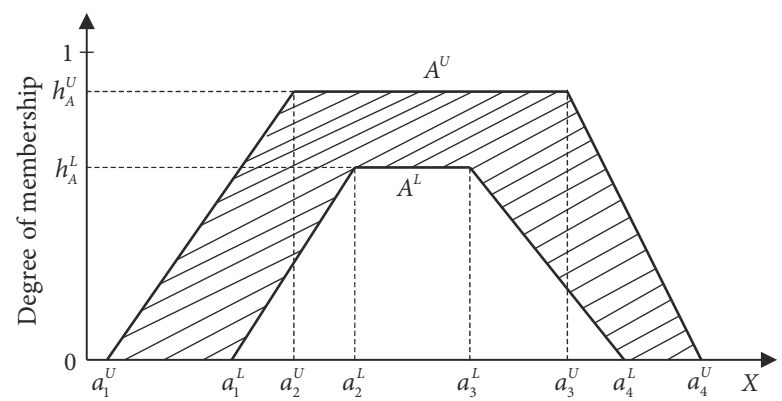

Fig. 1. A geometrical interpretation of an IT2TrF number $A$ on $X$

sic arithmetic operations on $A$ and $B$ are defined as follows (Chen 2012; Zhang, Z., Zhang, S. 2013):

$$
\begin{gathered}
A \oplus B=\left[\left(a_{1}^{L}+b_{1}^{L}, a_{2}^{L}+b_{2}^{L}, a_{3}^{L}+b_{3}^{L}, a_{4}^{L}+b_{4}^{L} ; \min \left\{h_{A}^{L}, h_{B}^{L}\right\}\right),\right. \\
\left.\left(a_{1}^{U}+b_{1}^{U}, a_{2}^{U}+b_{2}^{U}, a_{3}^{U}+b_{3}^{U}, a_{4}^{U}+b_{4}^{U} ; \min \left\{h_{A}^{U}, h_{B}^{U}\right\}\right)\right] ; \\
A \otimes B=\left[\left(a_{1}^{L} \times b_{1}^{L}, a_{2}^{L} \times b_{2}^{L}, a_{3}^{L} \times b_{3}^{L}, a_{4}^{L} \times b_{4}^{L} ; \min \left\{h_{A}^{L}, h_{B}^{L}\right\}\right),\right. \\
\left.\left(a_{1}^{U} \times b_{1}^{U}, a_{2}^{U} \times b_{2}^{U}, a_{3}^{U} \times b_{3}^{U}, a_{4}^{U} \times b_{4}^{U} ; \min \left\{h_{A}^{U}, h_{B}^{U}\right\}\right)\right] ; \\
A \varnothing B=\left[\left(a_{1}^{L} / b_{4}^{L}, a_{2}^{L} / b_{3}^{L}, a_{3}^{L} / b_{2}^{L}, a_{4}^{L} / b_{1}^{L} ; \min h_{A}^{L}, h_{B}^{L}\right\}\right),\left(a_{1}^{U} / b_{4}^{U}, a_{2}^{U} / b_{3}^{U},\right. \\
\left.\left.a_{3}^{U} / b_{2}^{U}, a_{4}^{U} / b_{1}^{U} ; \min \left\{h_{A}^{U}, h_{B}^{U}\right\}\right)\right], b_{1}^{L}, b_{2}^{L}, b_{3}^{L}, b_{4}^{L}, b_{1}^{U}, b_{2}^{U}, b_{3}^{U}, b_{4}^{U}>0 ; \\
\tau \cdot A=\left\{\left(\tau \times a_{1}^{L}, \tau \times a_{2}^{L}, \tau \times a_{3}^{L}, \tau \times a_{4}^{L} ; h_{A}^{L}\right),\left(\tau \times a_{1}^{U}, \tau \times a_{2}^{U}, \tau \times a_{3}^{U}, \tau \times a_{4}^{U} ; h_{A}^{U}\right)\right] \text { if } \tau \geq 0, \\
{\left[\left(\tau \times a_{4}^{L}, \tau \times a_{3}^{L}, \tau \times a_{2}^{L}, \tau \times a_{1}^{L} ; h_{A}^{L}\right),\left(\tau \times a_{4}^{U}, \tau \times a_{3}^{U}, \tau \times a_{2}^{U}, \tau \times a_{1}^{U} ; h_{A}^{U}\right)\right] \text { if } \tau \leq 0 .}
\end{gathered}
$$

The multiplication and division operations produce approximate IT2 $\operatorname{TrF}$ numbers for simple computations. In addition, the inclusion relationship of $A$ and $B$ is defined as follows (Zhang, Z., Zhang, S. 2013): $A \subseteq B$ if and only if $a_{1}^{L} \leq b_{1}^{L}, a_{2}^{L} \leq b_{2}^{L}, a_{3}^{L} \leq b_{3}^{L}, a_{4}^{L} \leq b_{4}^{L}, a_{1}^{U} \leq$ $b_{1}^{U}, a_{2}^{U} \leq b_{2}^{U}, a_{3}^{U} \leq b_{3}^{U}, a_{4}^{U} \leq b_{4}^{U}, h_{A}^{L} \leq h_{B}^{L}$, and $h_{A}^{U} \leq h_{B}^{U} . A \supseteq B$ if and only if $B \subseteq A$.

\section{MCDA using prioritised IT2F aggregation operators}

This section first formulates a decision environment based on IT2F sets. Next, this section extends the prioritised aggregation operators that were originally introduced by Yager $(2004,2008)$ to the IT2F environment. Based on IT2TrF numbers, a procedure is presented for determining the priority-based weights, and a new concept of prioritised IT2F aggregation operators is proposed. Finally, the proposed prioritised IT2F aggregation operator is used to address MCDA problems in an IT2TrF context. 


\subsection{IT2F decision environment}

Consider the following MCDA problem in which the ratings of alternative evaluations are expressed as IT2F sets, and prioritisation relationships exist over the criteria. Define $Z=\left\{z_{1}, z_{2}, \cdots, z_{m}\right\}$ as the set of decision alternatives, where $m$ is the number of alternatives. Define $\left.X=x_{1}, x_{2}, \cdots, x_{n}\right\}$ as the criteria set that contains $n$ criteria by which the alternative performances are measured. The set $X$ can be partitioned into $\eta$ classes, $X^{1}=$ $\left\{x_{1}, x_{2}, \cdots, x_{n_{1}}\right\}, X^{2}=\left\{x_{n_{1}+1}, x_{n_{1}+2}, \cdots, x_{n_{1}+n_{2}}\right\}, \cdots$, and $\left.X^{\eta}=x_{n_{\eta-1}+1}, x_{n_{\eta-1}+2}, \cdots, x_{n}\right\}$ with a linear ordering $X^{1} \succ X^{2} \succ \cdots \succ X^{\eta}$, where $\eta \leq n, \cup_{\kappa=1}^{\eta} X^{\kappa}=X$, and $X^{\kappa} \cap X^{\kappa^{\prime}}=\varnothing$ for $\kappa \neq \kappa^{\prime}$. Let $n_{\kappa}$ represent the number of criteria in $X^{\kappa}$. In addition, let $x_{j}^{\kappa}\left(j=1,2, \cdots, n_{\kappa}\right)$ denote an element $x_{n_{\kappa-1}+j}$ in $X^{\kappa}$. Next, denote the set of criteria in the $\kappa$ th priority class as $X^{\kappa}=\left\{x_{1}^{\kappa}, x_{2}^{\kappa}, \cdots, x_{n}^{\kappa} \quad(\kappa=1,2, \cdots, \eta)\right.$, where $\sum_{\kappa=1}^{\eta} n_{\kappa}=n$. The prioritisation relationship between the classes $X^{\kappa}$ and $X^{\kappa^{\prime}}$ indicates that the criteria in class $X^{\kappa}$ have a higher priority than those in class $X^{\kappa^{\prime}}$ if $\kappa<\kappa^{\prime}$.

Linguistic ratings can be appropriately represented by IT2 TrF numbers to directly address the uncertainties in complex or ill-defined situations. This paper adopted the standards introduced by Chen $(2011,2012)$ and Chen et al. (2013) to convert the linguistic terms into IT2TrF numbers. Table 1 depicts the employed nine-point linguistic scales and the corresponding IT2TrF numbers that are bounded within $[0,1]$.

Table 1. Linguistic variables and their corresponding IT2TrF numbers

\begin{tabular}{ll}
\hline \multicolumn{1}{c}{ Linguistic terms } & \multicolumn{1}{c}{ Corresponding IT2TrF numbers } \\
\hline Extremely poor $(\mathrm{EP})$ & {$[(0.0,0.0,0.0,0.0 ; 1.0),(0.0,0.0,0.0,0.0 ; 1.0)]$} \\
\hline Very poor $(\mathrm{VP})$ & {$[(0.0075,0.0075,0.015,0.0525 ; 0.8),(0.0,0.0,0.02,0.07 ; 1.0)]$} \\
\hline Poor $(\mathrm{P})$ & {$[(0.0875,0.12,0.16,0.1825 ; 0.8),(0.04,0.10,0.18,0.23 ; 1.0)]$} \\
\hline Medium poor $(\mathrm{MP})$ & {$[(0.2325,0.255,0.325,0.3575 ; 0.8),(0.17,0.22,0.36,0.42 ; 1.0)]$} \\
\hline Fair $(\mathrm{F})$ & {$[(0.4025,0.4525,0.5375,0.5675 ; 0.8),(0.32,0.41,0.58,0.65 ; 1.0)]$} \\
\hline Medium good (MG) & {$[(0.65,0.6725,0.7575,0.79 ; 0.8),(0.58,0.63,0.80,0.86 ; 1.0)]$} \\
\hline Good $(\mathrm{G})$ & {$[(0.7825,0.815,0.885,0.9075 ; 0.8),(0.72,0.78,0.92,0.97 ; 1.0)]$} \\
\hline Very good (VG) & {$[(0.9475,0.985,0.9925,0.9925 ; 0.8),(0.93,0.98,1.0,1.0 ; 1.0)]$} \\
\hline Extremely good (EG) & {$[(1.0,1.0,1.0,1.0 ; 1.0),(1.0,1.0,1.0,1.0 ; 1.0)]$} \\
\hline
\end{tabular}

Let an IT2TrF number $A_{i j}$ denote the evaluative rating of alternative $z_{i} \in Z$ with respect to criterion $x_{i} \in X$, where $A_{i j}$ is expressed as the following:

$$
A_{i j}=\left[A_{i j}^{L}, A_{i j}^{U}\right]=\left[\left(a_{1 i j}^{L}, a_{2 i j}^{L}, a_{3 i j}^{L}, a_{4 i j}^{L} ; h_{i j}^{L}\right),\left(a_{1 i j}^{U}, a_{2 i j}^{U}, a_{3 i j}^{U}, a_{4 i j}^{U} ; h_{i j}^{U}\right)\right],
$$

and where $0 \leq a_{1 i j}^{L} \leq a_{2 i j}^{L} \leq a_{3 i j}^{L} \leq a_{4 i j}^{L} \leq 1,0 \leq a_{1 i j}^{U} \leq a_{2 i j}^{U} \leq a_{3 i j}^{U} \leq a_{4 i j}^{U} \leq 1, a_{1 i j}^{U} \leq a_{1 i j}^{L}, a_{4 i j}^{L} \leq a_{4 i j}^{U}$, $0<h_{i j}^{L} \leq h_{i j}^{U} \leq 1$, and $A_{i j}^{L} \subseteq A_{i j}^{U}$. Alternatively, consider criterion $x_{j}^{\kappa} \in X^{\kappa}$. Let an IT2 TrF number $A_{i j}^{\kappa}$ denote the evaluative rating of alternative $z_{i} \in Z$ with respect to criterion $x_{j}^{\kappa} \in X^{\kappa}$. The IT2TrF rating $A_{i j}^{\kappa}$ is expressed as the following: 


$$
A_{i j}^{\kappa}=\left[A_{i j}^{\kappa L}, A_{i j}^{\kappa U}\right]=\left[\left(a_{1 i j}^{\kappa L}, a_{2 i j}^{\kappa L}, a_{3 i j}^{\kappa L}, a_{4 i j}^{\kappa L} ; h_{i j}^{\kappa L}\right),\left(a_{1 i j}^{\kappa U}, a_{2 i j}^{\kappa U}, a_{3 i j}^{\kappa U}, a_{4 i j}^{\kappa U} ; h_{i j}^{\kappa U}\right)\right],
$$

where $0 \leq a_{1 i j}^{\kappa L} \leq a_{2 i j}^{\kappa L} \leq a_{3 i j}^{\kappa L} \leq a_{4 i j}^{\kappa L} \leq 1,0 \leq a_{1 i j}^{\kappa U} \leq a_{2 i j}^{\kappa U} \leq a_{3 i j}^{\kappa U} \leq a_{4 i j}^{\kappa U} \leq 1, a_{1 i j}^{\kappa U} \leq a_{1 i j}^{\kappa L}, a_{4 i j}^{\kappa L} \leq a_{4 i j}^{\kappa U}$, $0<h_{i j}^{\kappa L} \leq h_{i j}^{\kappa U} \leq 1$, and $A_{i j}^{\kappa L} \subseteq A_{i j}^{\kappa U}$.

\subsection{Determination of priority-based weights}

For an MCDA problem with prioritised criteria, it is crucial to determine the weight of each priority class based on the prioritisation of the criteria and to aggregate the evaluative ratings of the alternatives with respect to prioritised criteria. As suggested by Yager (2008) and $\mathrm{Yu}$ and $\mathrm{Xu}$ (2013), the priority-based weights are associated with a criterion that is dependent on the satisfaction (or performance) of the higher priority criteria by modelling the prioritisation among criteria. Following the rationale above, an IT2 TrF number $Q_{i}^{\kappa}$ is defined to synthesise all of the IT2TrF ratings of a specific alternative $z_{i} \in Z$ with respect to all of the criteria in the same class as $X^{\kappa}$. Next, the synthesised value $Q_{i}^{\kappa}$ can be employed to develop an IT2F prioritised aggregation operator based on IT2TrF numbers.

Definition 5. Denote an IT2TrF number $A_{i j}^{\kappa}$ as the evaluative rating of alternative $z_{i} \in Z$ with respect to criterion $x_{j}^{\kappa} \in X^{\kappa}$, where $i=1,2, \cdots, m, j=1,2, \cdots, n_{\kappa}$, and $\kappa=1,2, \cdots, \eta$. The synthesised value $Q_{i}^{\kappa}$ for each $z_{i}$ is defined as follows:

$$
Q_{i}^{\kappa}= \begin{cases}{[(1,1,1,1 ; 1),(1,1,1,1 ; 1)]} & \text { if } \kappa=0, \\ n_{\kappa} A_{i j}^{\kappa} & \text { if } \kappa=1,2, \cdots, \eta-1 .\end{cases}
$$

The synthesised value $Q_{i}^{\kappa}$ can be computed using the multiplication operation based on IT2TrF numbers as follows:

$$
Q_{i}^{\kappa}= \begin{cases}{[(1,1,1,1 ; 1),(1,1,1,1 ; 1)]} & \text { if } \kappa=0, \\ {\left[\prod_{j=1}^{n_{\kappa}} a_{1 i j}^{\kappa L}, \prod_{j=1}^{n_{\kappa}} a_{2 i j}^{\kappa L}, \prod_{j=1}^{n_{\kappa}} a_{3 i j}^{\kappa L}, \prod_{j=1}^{n_{\kappa}} a_{4 i j}^{\kappa L} ; \min _{j=1}^{n_{\kappa}} h_{i j}^{\kappa L}\right),} & \text { if } \kappa=1,2, \cdots, \eta-1 . \\ \left.\left(\prod_{j=1}^{n_{\kappa}} a_{1 i j}^{\kappa U}, \prod_{j=1}^{n_{\kappa}} a_{2 i j}^{\kappa U}, \prod_{j=1}^{n_{\kappa}} a_{3 i j}^{\kappa U}, \prod_{j=1}^{n_{\kappa}} a_{4 i j}^{\kappa U} ; \min _{j=1}^{n_{\kappa}} h_{i j}^{\kappa U}\right)\right] & \end{cases}
$$

For brevity, $Q_{i}^{\kappa}$ is denoted as:

$$
Q_{i}^{\kappa}=\left[Q_{i}^{\kappa L}, Q_{i}^{\kappa U}\right]=\left[\left(q_{1 i}^{\kappa L}, q_{2 i}^{\kappa L}, q_{3 i}^{\kappa L}, q_{4 i}^{\kappa L} ; h_{q i}^{\kappa L}\right),\left(q_{1 i}^{\kappa U}, q_{2 i}^{\kappa U}, q_{3 i}^{\kappa U}, q_{4 i}^{\kappa U} ; h_{q i}^{\kappa U}\right)\right]
$$

for $\kappa=0,1, \cdots, \eta-1$ and $i=1,2, \cdots, m$.

Definition 6. For alternative $z_{i} \in Z$, the priority-based weight $W_{i}^{\kappa}$ of the $\kappa$ th class is defined by means of $Q_{i}^{\kappa}$, as follows:

$$
W_{i}^{\kappa}=\bigotimes_{\varphi=1}^{\kappa} Q_{i}^{\varphi-1} \quad \kappa=1,2, \cdots, \eta \text { and } i=1,2, \cdots, m .
$$


The priority-based weight $W_{i}^{\kappa}$ can be computed using the multiplication operation of IT2TrF numbers as follows:

$$
\begin{aligned}
W_{i}^{\kappa}= & {\left[\left(\prod_{\varphi=1}^{\kappa} q_{1 i}^{\varphi-1, L}, \prod_{\varphi=1}^{\kappa} q_{2 i}^{\varphi-1, L}, \prod_{\varphi=1}^{\kappa} q_{3 i}^{\varphi-1, L}, \prod_{\varphi=1}^{\kappa} q_{4 i}^{\varphi-1, L} ; \min _{\varphi=1}^{\kappa} h_{q i}^{\varphi-1, L}\right),\right.} \\
& \left.\left(\prod_{\varphi=1}^{\kappa} q_{1 i}^{\varphi-1, U}, \prod_{\varphi=1}^{\kappa} q_{2 i}^{\varphi-1, U}, \prod_{\varphi=1}^{\kappa} q_{3 i}^{\varphi-1, U}, \prod_{\varphi=1}^{\kappa} q_{4 i}^{\varphi-1, U} ; \min _{\varphi=1}^{\kappa} h_{q i}^{\varphi-1, U}\right)\right] .
\end{aligned}
$$

For brevity, $W_{i}^{\kappa}$ is denoted as:

$$
W_{i}^{\kappa}=\left[W_{i}^{\kappa L}, W_{i}^{\kappa U}\right]=\left[\left(w_{1 i}^{\kappa L}, w_{2 i}^{\kappa L}, w_{3 i}^{\kappa L}, w_{4 i}^{\kappa L} ; h_{w i}^{\kappa L}\right),\left(w_{1 i}^{\kappa U}, w_{2 i}^{\kappa U}, w_{3 i}^{\kappa U}, w_{4 i}^{\kappa U} ; h_{w i}^{\kappa U}\right)\right],
$$

for $\kappa=1,2, \cdots, \eta$ and $i=1,2, \cdots, m$.

Theorem 1. Let $A_{i j}^{\kappa}\left(=\left[A_{i j}^{\kappa L}, A_{i j}^{\kappa U}\right]=\left[\left(a_{1 i j}^{\kappa L}, a_{2 i j}^{\kappa L}, a_{3 i j}^{\kappa L}, a_{4 i j}^{\kappa L} ; h_{i j}^{\kappa L}\right),\left(a_{1 i j}^{\kappa U}, a_{2 i j}^{\kappa U}, a_{3 i j}^{\kappa U}, a_{4 i j}^{\kappa U} ; h_{i j}^{\kappa U}\right)\right]\right)$ $\left(i=1,2, \cdots, m, j=1,2, \cdots, n_{\kappa}\right.$, and $\left.\kappa=1,2, \cdots, \eta\right)$ be a collection of IT2TrF numbers that are bounded within $[0,1]$. The synthesised value $Q_{i}^{\kappa}(\kappa=0,1, \cdots, \eta-1)$ and the priority-based weight $W_{i}^{\kappa}(\kappa=1,2, \cdots, \eta)$ for all $i$ are also IT2TrF numbers bounded within $[0,1]$.

Proof. See Appendix A.

Note that the priority-based weights of the criteria with higher priority dominate those of the lower prior criteria (see Theorem 2). Furthermore, the priority-based weights that correspond to distinct priority classes are usually different among the $m$ alternatives.

Theorem 2. The priority-based weights for alternative $z_{i} \in Z$ satisfy $W_{i}^{\kappa} \supseteq W_{i}^{\kappa "}$ if $\kappa<\kappa "$, where $\kappa, \kappa^{\prime \prime}=1,2, \cdots, \eta$ and $\kappa \neq \kappa^{\prime \prime}$.

Proof. See Appendix B.

For alternative $z_{i} \in Z$, the normalised priority-based weight $W_{i}^{\prime \kappa}$ of the $\kappa$ th class is computed by the following:

$$
\left.W_{i}^{\prime \kappa}=W_{i}^{\kappa} \varnothing\left(\bigoplus_{\gamma=1}^{\eta} n_{\gamma} \cdot W_{i}^{\gamma}\right)\right) \kappa=1,2, \cdots, \eta \text { and } i=1,2, \cdots, m,
$$

where the following is obtained using the addition and multiplication operations:

$$
\begin{aligned}
\underset{\gamma=1}{\oplus}\left(n_{\gamma} \cdot W_{i}^{\gamma}=\right. & {\left[\left(\sum_{\gamma=1}^{\eta} n_{\gamma} \cdot w_{1 i}^{\gamma L}, \sum_{\gamma=1}^{\eta} n_{\gamma} \cdot w_{2 i}^{\gamma L}, \sum_{\gamma=1}^{\eta} n_{\gamma} \cdot w_{3 i}^{\gamma L}, \sum_{\gamma=1}^{\eta} n_{\gamma} \cdot w_{4 i}^{\gamma L} ; \min _{\gamma=1}^{\eta} h_{w i}^{\gamma L}\right),\right.} \\
& \left.\left(\sum_{\gamma=1}^{\eta} n_{\gamma} \cdot w_{1 i}^{\gamma U}, \sum_{\gamma=1}^{\eta} n_{\gamma} \cdot w_{2 i}^{\gamma U}, \sum_{\gamma=1}^{\eta} n_{\gamma} \cdot w_{3 i}^{\gamma U}, \sum_{\gamma=1}^{\eta} n_{\gamma} \cdot w_{4 i}^{\gamma U} ; \min _{\gamma=1}^{\eta} h_{w i}^{\gamma U}\right)\right] .
\end{aligned}
$$

Let $\xi \in\{1,2,3,4\}$ and denote $w_{\xi i}^{\prime \kappa L}=w_{\xi i}^{\kappa L} / \sum_{\gamma=1}^{\eta}\left(n_{\gamma} \cdot w_{(5-\xi) i}^{\gamma L}\right), w_{\xi i}^{\prime \kappa U}=w_{\xi i}^{\kappa U} / \sum_{\gamma=1}^{\eta}\left(n_{\gamma} \cdot w_{(5-\xi) i}^{\gamma U}\right.$, $\left.\begin{array}{l}h_{w^{\prime} i}^{\kappa L}=\min \\ \text { noted as }\end{array} h_{w i}^{\kappa L}, \min _{\gamma=1}^{\eta} h_{w i}^{\gamma L}\right\}$, and $h_{w^{\prime} i}^{\kappa U}=\min \left\{h_{w i}^{\kappa U}, \min _{\gamma=1}^{\eta} h_{w i}^{\gamma U}\right\}$ for each $\xi$. Next, $W_{i}^{\prime \kappa}$ is de- 


$$
W_{i}^{\prime \kappa}=\left[W_{i}^{\prime \kappa L}, W_{i}^{\prime \kappa U}\right]=\left[\left(w_{1 i}^{\prime \kappa L}, w_{2 i}^{\prime \kappa L}, w_{3 i}^{\prime \kappa L}, w_{4 i}^{\prime \kappa L} ; h_{w^{\prime} i}^{\kappa L}\right),\left(w_{1 i}^{\prime \kappa U}, w_{2 i}^{\prime \kappa U}, w_{3 i}^{\prime \kappa U}, w_{4 i}^{\prime \kappa U} ; h_{w^{\prime} i}^{\kappa U}\right)\right]
$$

for $\kappa=1,2, \cdots, \eta$ and $i=1,2, \cdots, m$.

\section{Theorem 3}

Let $W_{i}^{\kappa}\left(=\left[W_{i}^{\kappa L}, W_{i}^{\kappa U}\right]=\left[\left(w_{1 i}^{\kappa L}, w_{2 i}^{\kappa L}, w_{3 i}^{\kappa L}, w_{4 i}^{\kappa L} ; h_{w i}^{\kappa L}\right),\left(w_{1 i}^{\kappa U}, w_{2 i}^{\kappa U}, w_{3 i}^{\kappa U}, w_{4 i}^{\kappa U} ; h_{w i}^{\kappa U}\right)\right]\right)$ $(i=1,2, \cdots, m$ and $\kappa=1,2, \cdots, \eta)$ be the priority-based weight expressed by an IT2TrF number bounded within $[0,1]$. The normalised value $W_{i}^{\prime \kappa}(\kappa=1,2, \cdots, \eta)$ for all $i$ and $\kappa$ is also an IT2TrF number bounded within $[0,1]$.

Proof. See Appendix C.

Theorem 4. The normalised priority-based weights for alternative $z_{i} \in Z$ satisfy $W_{i}^{\prime \kappa} \supseteq W_{i}^{\prime \kappa}$ if $\kappa<\kappa^{\prime \prime}$, where $\kappa, \kappa^{\prime \prime}=1,2, \cdots, \eta$ and $\kappa \neq \kappa^{\prime \prime}$.

Proof. The proof of this theorem is similar to that of Theorem 2.

It should be noted that $\oplus_{\kappa=1} n \cdot W_{i}^{\prime} \neq[1,1,1,1 ; 1,(1,1,1,1 ; 1]$ in the context of IT2F sets. Observe that:

$$
\begin{aligned}
& \oplus_{\kappa=1}^{\eta}\left(n_{\kappa} \cdot w_{i}^{\prime \kappa}\right)=\left[\left(\frac{\sum_{\kappa=1}^{\eta} n_{\kappa} \cdot w_{1 i}^{\kappa L}}{\sum_{\kappa=1}^{\eta} n_{\kappa} \cdot w_{4 i}^{\kappa L}}, \frac{\sum_{\kappa=1}^{\eta} n_{\kappa} \cdot w_{2 i}^{\kappa L}}{\sum_{\kappa=1}^{\eta} n_{\kappa} \cdot w_{3 i}^{\kappa L}}, \frac{\sum_{\kappa=1}^{\eta} n_{\kappa} \cdot w_{3 i}^{\kappa L}}{\sum_{\kappa=1}^{\eta} n_{\kappa} \cdot w_{2 i}^{\kappa L}}, \frac{\sum_{\kappa=1}^{\eta} n_{\kappa} \cdot w_{4 i}^{\kappa L}}{\sum_{\kappa=1}^{\eta} n_{\kappa} \cdot w_{1 i}^{\kappa L}} ; \min _{\kappa=1}^{\eta} h_{w i}^{\kappa L}\right]\right. \\
& \left.\left(\frac{\sum_{\kappa=1}^{\eta} n_{\kappa} \cdot w_{1 i}^{\kappa U}}{\sum_{\kappa=1}^{\eta} n_{\kappa} \cdot w_{4 i}^{\kappa U}}, \frac{\sum_{\kappa=1}^{\eta} n_{\kappa} \cdot w_{2 i}^{\kappa U}}{\sum_{\kappa=1}^{\eta} n_{\kappa} \cdot w_{3 i}^{\kappa U}}, \frac{\sum_{\kappa=1}^{\eta} n_{\kappa} \cdot w_{3 i}^{\kappa U}}{\sum_{\kappa=1}^{\eta} n_{\kappa} \cdot w_{2 i}^{\kappa U}}, \frac{\sum_{\kappa=1}^{\eta} n_{\kappa} \cdot w_{4 i}^{\kappa U}}{\sum_{\kappa=1}^{\eta} n_{\kappa} \cdot w_{1 i}^{\kappa U}} ; \min _{\kappa=1}^{\eta} h_{w i}^{\kappa U}\right)\right] .
\end{aligned}
$$

It is obvious that the sum of the normalised priority-based weights will not be restricted to $[(1,1,1,1 ; 1),(1,1,1,1 ; 1)]$. However, $\oplus_{\kappa=1}^{\eta}\left(n_{\kappa} \cdot W_{i}^{\prime \kappa}\right)$ will approach $[(1,1,1,1 ; 1),(1,1,1,1 ; 1)]$ in most situations.

\subsection{A prioritised IT2F aggregation operator}

This subsection introduces a new concept of prioritised IT2F aggregation operators. As stated previously, the IT2 TrF number $A_{i j}$ is denoted as the evaluative rating of alternative $z_{i} \in Z$ with respect to criterion $x_{i} \in X$. For convenience, let $\Omega$ be the set of all IT2 $\operatorname{TrF}$ numbers. The IT2TrF ratings can be aggregated for each alternative $z_{i}$ using the following prioritised IT2F aggregation operator, as follows.

Definition 7. Denote an IT2TrF number $A_{i j}$ as the evaluative rating of alternative $z_{i} \in Z$ with respect to criterion $x_{i} \in X$, where $i=1,2, \cdots, m$ and $j=1,2, \cdots, n$. For $i=1,2, \cdots, m$, let PIT2FA: $\Omega^{n} \rightarrow \Omega$; if:

$$
\operatorname{PIT} 2 F A\left(A_{i 1}, A_{i 2}, \cdots, A_{i n}\right)=\bigoplus_{\kappa=1}^{\eta}\left(\bigoplus_{j=1}^{n_{\kappa}}\left(W_{i}^{\prime \kappa} \otimes A_{i j}^{\kappa}\right)\right),
$$


and the function PIT2FA is referred to as a prioritised IT2F aggregation operator, where the normalised priority-based weight $W_{i}^{\prime \kappa}\left(=\left[\left(w_{1 i}^{\prime \kappa L}, w_{2 i}^{\prime \kappa L}, w_{3 i}^{\prime \kappa L}, w_{4 i}^{\prime \kappa L} ; h_{w^{\prime} i}^{\kappa L}\right),\left(w_{1 i}^{\prime \kappa U}, w_{2 i}^{\prime \kappa U}, w_{3 i}^{\prime \kappa U}\right.\right.\right.$, $\left.\left.\left.w_{4 i}^{\prime \kappa U} ; h_{w^{\prime} i}^{\kappa U}\right)\right]\right)$ and the rating $A_{i j}^{\kappa}\left(=\left[\left(a_{1 i j}^{\kappa L}, a_{2 i j}^{\kappa L}, a_{3 i j}^{\kappa L}, a_{4 i j}^{\kappa L} ; h_{i j}^{\kappa L}\right),\left(a_{1 i j}^{\kappa U}, a_{2 i j}^{\kappa U}, a_{3 i j}^{\kappa U}, a_{4 i j}^{\kappa U} ; h_{i j}^{\kappa U}\right)\right]\right)$ are IT2TrF numbers bounded within $[\overline{0}, 1]$.

Theorem 5. Let $A_{i j}^{\kappa}\left(=\left[\left(a_{1 i j}^{\kappa L}, a_{2 i j}^{\kappa L}, a_{3 i j}^{\kappa L}, a_{4 i j}^{\kappa L} ; h_{i j}^{\kappa L}\right),\left(a_{1 i j}^{\kappa U}, a_{2 i j}^{\kappa U}, a_{3 i j}^{\kappa U}, a_{4 i j}^{\kappa U} ; h_{i j}^{\kappa U}\right)\right]\right)(i=1,2, \cdots, m$, $j=1,2, \cdots, n_{\kappa}$, and $\left.\kappa=1,2, \cdots, \eta\right)$ be a collection of IT2 TrF numbers bounded within $[0,1]$. Let $W_{i}^{\prime \kappa} \quad\left(=\left[\left(w_{1 i}^{\prime \kappa L}, w_{2 i}^{\prime \kappa L}, w_{3 i}^{\prime \kappa L}, w_{4 i}^{\prime \kappa L} ; h_{w^{\prime} i}^{\kappa L}\right),\left(w_{1 i}^{\prime \kappa U}, w_{2 i}^{\prime \kappa U}, w_{3 i}^{\prime \kappa U}, w_{4 i}^{\prime \kappa U} ; h_{w^{\prime} i}^{\kappa}\right)\right]\right) \quad(i=1,2, \cdots, m$ and $\kappa=1,2, \cdots, \eta)$ be the normalised priority-based weight. Next, the synthetic evaluation $\operatorname{PIT} 2 F A\left(A_{i 1}, A_{i 2}, \cdots, A_{i n}\right)$ for all $i$ is also an IT2TrF number.

Proof. See Appendix D.

$\operatorname{PIT} 2 F A\left(A_{i 1}, A_{i 2}, \cdots, A_{i n}\right)$ represents the synthetic evaluation of alternative $z_{i} \in$ $Z$ in terms of all of the prioritised criteria. Let $\xi \in\{1,2,3,4\}$. For brevity, denote $p_{\xi i}^{L}=\sum_{\kappa=1}^{\eta} \sum_{j=1}^{n_{\kappa}} w_{\xi i}^{\prime \kappa L} \cdot a_{\xi i j}^{\kappa L}, \quad p_{\xi i}^{U}=\sum_{\kappa=1}^{\eta} \sum_{j=1}^{n_{\kappa}} w_{\xi i}^{\prime \kappa U} \cdot a_{\xi i j}^{\kappa U}, \quad h_{p i}^{L}=\min _{\kappa=1}^{\eta} \min _{j=1}^{n_{\kappa}}\left(\min \left\{h_{w^{\prime} i}^{\kappa L}, h_{i j}^{\kappa L}\right\}\right), \quad$ and $h_{p i}^{U}=\min _{\kappa=1}^{\eta} \min _{j=1}^{n_{\kappa}}\left(\min \left\{h_{w^{\prime} i}^{\kappa U}, h_{i j}^{\kappa U}\right\}\right)$ for each $\xi$. Therefore, PIT $2 F A\left(A_{i 1}, A_{i 2}, \cdots, A_{i n}\right)$ is denoted as

$$
\operatorname{PIT} 2 F A\left(A_{i 1}, A_{i 2}, \cdots, A_{i n}\right)=\left[P_{i}^{L}, P_{i}^{U}\right]=\left[\left(p_{1 i}^{L}, p_{2 i}^{L}, p_{3 i}^{L}, p_{4 i}^{L} ; h_{p i}^{L}\right),\left(p_{1 i}^{U}, p_{2 i}^{U}, p_{3 i}^{U}, p_{4 i}^{U} ; h_{p i}^{U}\right)\right],
$$

where $\quad 0 \leq p_{1 i}^{L} \leq p_{2 i}^{L} \leq p_{3 i}^{L} \leq p_{4 i}^{L}, \quad 0 \leq p_{1 i}^{U} \leq p_{2 i}^{U} \leq p_{3 i}^{U} \leq p_{4 i}^{U}, \quad p_{1 i}^{U} \leq p_{1 i}^{L}, \quad p_{4 i}^{L} \leq p_{4 i}^{U}, \quad 0<h_{p i}^{L} \leq$ $h_{p i}^{U} \leq 1$, and $P_{i}^{L} \subseteq P_{i}^{U}$.

This paper uses the IT2 TrF weights instead of scalar weights to express the relative importance of various priority classes. Additionally, the sum of the normalised priority-based weights is not equal to $[(1,1,1,1 ; 1),(1,1,1,1 ; 1)]$. Therefore, as indicated in Theorem 5 , the synthetic evaluation $\operatorname{PIT} 2 F A\left(A_{i 1}, A_{i 2}, \cdots, A_{\text {in }}\right)$ is an IT2TrF number, but it might be not bounded within $[0,1]$. In addition, the developed prioritised IT2F aggregation operator does not satisfy the properties of idempotency and boundary conditions in general. However, the prioritised IT2F aggregation operator still possesses the property of monotonicity.

\section{Theorem 6}

Let $A_{i j}^{\kappa}\left(=\left[\left(a_{1 i j}^{\kappa L}, a_{2 i j}^{\kappa L}, a_{3 i j}^{\kappa L}, a_{4 i j}^{\kappa L} ; h_{i j}^{\kappa L}\right),\left(a_{1 i j}^{\kappa U}, a_{2 i j}^{\kappa U}, a_{3 i j}^{\kappa U}, a_{4 i j}^{\kappa U} ; h_{i j}^{\kappa U}\right)\right]\right)$

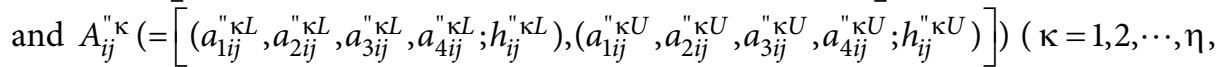
$i=1,2, \cdots, m$, and $\left.j=1,2, \cdots, n_{\kappa}\right)$ be two collections of IT2TrF numbers.

Let $W_{i}^{\prime \kappa}\left(=\left[\left(w_{1 i}^{\prime \kappa L}, w_{2 i}^{\prime \kappa L}, w_{3 i}^{\prime \kappa L}, w_{4 i}^{\prime \kappa L} ; h_{w^{\prime} i}^{\kappa L}\right),\left(w_{1 i}^{\prime \kappa U}, w_{2 i}^{\prime \kappa U}, w_{3 i}^{\prime \kappa U}, w_{4 i}^{\prime \kappa U} ; h_{w^{\prime} i}^{\kappa U}\right)\right]\right)(\kappa=1,2, \cdots, \eta$ and $i=1,2, \cdots, m)$ be the normalised priority-based weight. If $A_{i j}^{\kappa} \subseteq A_{i j}^{\prime \kappa}$ for all $\kappa, i$, and $j$, then

$$
\operatorname{PIT} 2 F A\left(A_{i 1}, A_{i 2}, \cdots, A_{i n}\right) \subseteq \operatorname{PIT} 2 F A\left(A_{i 1}^{\prime \prime}, A_{i 2}^{\prime \prime}, \cdots, A_{i n}^{\prime \prime}\right) \quad i=1,2, \cdots, m .
$$

Proof. See Appendix E. 


\subsection{MCDA method using prioritised IT2F aggregation operators}

To compare the synthetic evaluation $\operatorname{PIT} 2 F A\left(A_{i 1}, A_{i 2}, \cdots, A_{i n}\right)$ of the alternative $z_{i} \in Z$, this paper uses an approach that uses signed distances to convert the IT2TrF number of $\operatorname{PIT} 2 F A\left(A_{i 1}, A_{i 2}, \cdots, A_{i n}\right)$ into real numbers. More specifically, the signed distances in the context of IT2F sets (Chen 2012) are used to define the ordering of the IT2TrF numbers. Let the level 1 fuzzy number $\tilde{0}_{1}$ map onto the vertical axis at the origin. For each $z_{i} \in Z$, the signed distance from $\operatorname{PIT} 2 F A\left(A_{i 1}, A_{i 2}, \cdots, A_{i n}\right)$ to $\tilde{0}_{1}$ is computed as follows:

$$
\begin{aligned}
& d\left(\operatorname{PIT} 2 F A\left(A_{i 1}, A_{i 2}, \cdots, A_{i n}\right), \tilde{0}_{1}\right)= \\
& \frac{1}{8}\left(p_{1 i}^{L}+p_{2 i}^{L}+p_{3 i}^{L}+p_{4 i}^{L}+4 p_{1 i}^{U}+2 p_{2 i}^{U}+2 p_{3 i}^{U}+4 p_{4 i}^{U}+3\left(p_{2 i}^{U}+p_{3 i}^{U}-p_{1 i}^{U}-p_{4 i}^{U}\right) \frac{h_{p i}^{L}}{h_{p i}^{U}}\right) .
\end{aligned}
$$

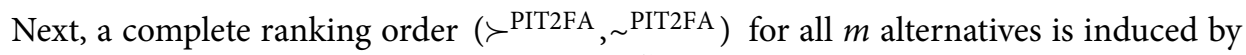
the signed distance $d\left(\operatorname{PIT} 2 F A\left(A_{i 1}, A_{i 2}, \cdots, A_{i n}\right), \tilde{0}_{1}\right)$, as follows:

$$
\left\{\begin{array}{lr}
z_{i_{1}} \succ^{\text {PIT2FA }} z_{i_{2}} & \text { if and only if } d\left(\operatorname{PIT} 2 F A\left(A_{i_{1} 1}, A_{i_{1} 2}, \cdots, A_{i_{1} n}\right), \tilde{0}_{1}\right) \\
\left(z_{i_{1}} \text { outranks } z_{i_{2}}\right) & >d\left(\operatorname{PIT} 2 F A\left(A_{i_{2} 1}, A_{i_{2} 2}, \cdots, A_{i_{2} n}\right), \tilde{0}_{1}\right) \\
z_{i_{1}} \sim^{\text {PIT2FA }} z_{i_{2}} & \text { if and only if } d\left(\operatorname{PIT} 2 F A\left(A_{i_{1} 1}, A_{i_{1} 2}, \cdots, A_{i_{1} n}\right), \tilde{0}_{1}\right) \\
\left(z_{i_{1}} \text { is indifferent to } z_{i_{2}}\right) & =d\left(\operatorname{PIT} 2 F A\left(A_{i_{2} 1}, A_{i_{2} 2}, \cdots, A_{i_{2} n}\right), \tilde{0}_{1}\right)
\end{array}\right.
$$

The prioritised IT2F aggregation operator-based approach for solving an MCDA problem in the IT2TrFN environment is summarised in the following steps:

Step 1: Formulate an MCDA problem with prioritised criteria. Specify the alternative set $Z=\left\{z_{1}, z_{2}, \cdots, z_{m}\right\}$ and the criteria set $X=\left\{x_{1}, x_{2}, \cdots, x_{n}\right\}$.

Step 2: Designate the prioritisation relationships among the $n$ criteria. Next, the criteria set $X$ is divided into $\eta$ priority classes $X^{1}, X^{2}, \cdots$, and $X^{\eta}$, where $X^{\kappa}=\left\{x_{1}^{\kappa}, x_{2}^{\kappa}, \cdots, x_{n}^{\kappa}\right.$ $(\kappa=1,2, \cdots, \eta)$.

Step 3: Select appropriate linguistic variables (e.g., Table 1) or other data collection tools to establish the IT2TrF rating $A_{i j}^{\kappa}$ in (8) for alternative $z_{i} \in Z$ with respect to criteria $x_{j}^{\kappa} \in X^{\kappa}$, which are provided by the decision-maker.

Step 4: Apply (10) to calculate the synthesised value $Q_{i}^{\kappa}$ of alternative $z_{i} \in Z$ in the $\kappa$ th priority class.

Step 5: Use (13) and (15) to compute the priority-based weight $W_{i}^{\kappa}$ and the normalised priority-based weight $W_{i}^{\prime \kappa}$, respectively, of the $\kappa$ th priority class for each alternative $z_{i} \in Z$.

Step 6: Aggregate the individual IT2TrF ratings using the prioritised IT2F aggregation operator in (19) to obtain PIT2FA $\left(A_{i 1}, A_{i 2}, \cdots, A_{i n}\right)$ of alternative $z_{i} \in Z$.

Step 7: Compute the signed distance $d\left(\operatorname{PIT} 2 F A\left(A_{i 1}, A_{i 2}, \cdots, A_{i n}\right), \tilde{0}_{1}\right)$ for each alternative $z_{i} \in Z$ using (22). Next, determine the complete ranking order for the set $Z$ of alternatives using ( $\left.\succ^{\mathrm{PIT} 2 \mathrm{FA}}, \sim^{\mathrm{PIT} 2 \mathrm{FA}}\right)$ in (23). 


\section{Illustrative applications}

This section explores a practical example of a landfill site selection problem to demonstrate the effectiveness of the prioritised IT2F aggregation operator-based method for MCDA in an IT2TrFN framework.

This practical example involves a problem that addresses the selection of landfill sites in a city. The criteria that must be considered when selecting a landfill site are complicated because the interests and rights of the stakeholders and the general public must be considered. Therefore, according to the city government requirements, the stakeholders, public representatives, and city officers have proposed several evaluation criteria for the landfill site selection process. The details of these criteria are outlined in Table 2.

Table 2. Criteria used to evaluate the landfill sites.

\begin{tabular}{|c|c|}
\hline Criterion $\left(x_{j}\right)$ & Explanation \\
\hline $\begin{array}{l}\text { Environmental } \\
\text { impact }\left(x_{1}\right)\end{array}$ & $\begin{array}{l}\text { The process of constructing a landfill could destroy groundwater protection areas. } \\
\text { Future operations of the landfill could also have a negative impact on the soil and } \\
\text { geology. Without proper consideration of environmental factors, the residents near } \\
\text { the landfill site (or the general public) who use the water resources will be placed } \\
\text { at risk, and their health will be threatened. }\end{array}$ \\
\hline $\begin{array}{l}\text { Ecological } \\
\text { impact }\left(x_{2}\right)\end{array}$ & $\begin{array}{l}\text { It is difficult to preserve the ecology. It is especially difficult to preserve protected } \\
\text { areas because they are vulnerable to damage from the surrounding environment, } \\
\text { and this damage can affect the ecosystem. Therefore, a landfill should not be } \\
\text { constructed in national parks, wetlands, or the surroundings of ecological or } \\
\text { animal protection areas. }\end{array}$ \\
\hline $\begin{array}{l}\text { Terrain } \\
\text { suitability }\left(x_{3}\right)\end{array}$ & $\begin{array}{l}\text { The suitability of the terrain in the selected location is determined by the slope of } \\
\text { the terrain and the altitude of the location. If the slope of the terrain is too steep, } \\
\text { then constructing a landfill on this site will easily increase the external pollution. } \\
\text { The best slope should be less than } 12 \% \text {, to prevent pollution from flowing out of } \\
\text { the landfill. }\end{array}$ \\
\hline $\begin{array}{l}\text { Transportation } \\
\text { convenience }\left(x_{4}\right)\end{array}$ & $\begin{array}{l}\text { If the site is located in a remote location, then the lack of transportation } \\
\text { infrastructure and the high costs of transporting the garbage to the landfill site will } \\
\text { be a source of inefficiency in the entire landfill process. Therefore, the convenience } \\
\text { of the transportation network near the landfill site must be considered. }\end{array}$ \\
\hline $\begin{array}{l}\text { Construction } \\
\text { cost }\left(x_{5}\right)\end{array}$ & $\begin{array}{l}\text { The construction costs include the cost of the land, the compensation to local } \\
\text { residents, and the costs of the landfill operations and management. The construction } \\
\text { of the landfill will also affect the value of the surrounding land and its agricultural } \\
\text { productivity. These costs also should be included in the construction costs. }\end{array}$ \\
\hline $\begin{array}{l}\text { Community } \\
\text { equity }\left(x_{6}\right)\end{array}$ & $\begin{array}{l}\text { To successfully construct the landfill, the community must fairly share the risks } \\
\text { that are involved in the process. In other words, the risk that is attributed to } \\
\text { establishment of the landfill site should be shared equally by the surrounding } \\
\text { communities; it should not be borne by a small number of people who then lose } \\
\text { their equity. }\end{array}$ \\
\hline $\begin{array}{l}\text { Historic impact } \\
\left(x_{7}\right)\end{array}$ & $\begin{array}{l}\text { The historic impact includes, e.g., the damage to the aesthetics of the location } \\
\text { and the diffusion of strange smells from the landfill site. Although not hazardous } \\
\text { to human health, these effects will influence the public perception of the site and } \\
\text { could even be viewed by the public as a symbol of the land. These effects could } \\
\text { influence the sightseeing or tourist attractions that surround the land. }\end{array}$ \\
\hline
\end{tabular}


The computational procedure of the proposed prioritised IT2F aggregation operator-based method is summarised as follows. In Step 1, four candidate landfill sites are proposed in the MCDA problem; the set of all of the candidate sites is denoted by $Z=\left\{z_{1}, z_{2}, z_{3}, z_{4}\right\}$. As stated in Table 2 , the set of evaluative criteria is denoted by $X=\left\{x_{1}, x_{2}, \cdots, x_{8}\right\}$.

In Step 2, the governing authority provides the prioritisation relationships among the criteria (see Table 3 ) in which $x_{1}$ and $x_{2}$ belong to the first priority level, $x_{3}$ and $x_{4}$ belong to the second priority level, $x_{5}$ and $x_{6}$ belong to the third priority level, and $x_{7}$ belongs to the last priority level. Next, the set $X$ of criteria can be partitioned into four distinct classes, $X^{1}$, $X^{2}, X^{3}$, and $X^{4}$, such that $X^{1}=\left\{x_{1}^{1}, x_{2}^{1}\right\}, X^{2}=\left\{x_{1}^{2}, x_{2}^{2}\right\}, X^{3}=\left\{x_{1}^{3}, x_{2}^{3}\right\}$, and $X^{4}=\left\{x_{1}^{4}\right\}$. In this work, $n_{1}=2, n_{2}=2, n_{3}=2, n_{4}=1, \eta=4$, and $\sum_{\kappa=1}^{4} n_{\kappa}=7$. The prioritisation among these classes is $X^{1} \succ X^{2} \succ X^{3} \succ X^{4}$.

Table 3. Prioritisation relationships and linguistic ratings

\begin{tabular}{llcccc}
\hline \multirow{2}{*}{ Prioritised criterion $\left(x_{j}^{\mathrm{K}}\right)$} & Priority level & \multicolumn{4}{c}{ Candidate locations } \\
\cline { 3 - 7 } & & $z_{1}$ & $z_{2}$ & $z_{3}$ & $z_{4}$ \\
\hline Environmental impact $\left(x_{1}^{1}\right)$ & First priority & $\mathrm{G}$ & $\mathrm{F}$ & $\mathrm{P}$ & $\mathrm{P}$ \\
\hline Ecological impact $\left(x_{2}^{1}\right)$ & First priority & $\mathrm{G}$ & $\mathrm{MG}$ & $\mathrm{P}$ & $\mathrm{MP}$ \\
\hline Terrain suitability $\left(x_{1}^{2}\right)$ & Second priority & EG & VG & VP & $\mathrm{P}$ \\
\hline Transportation convenience $\left(x_{2}^{2}\right)$ & Second priority & $\mathrm{P}$ & $\mathrm{VP}$ & $\mathrm{EG}$ & $\mathrm{G}$ \\
\hline Construction cost $\left(x_{1}^{3}\right)$ & Third priority & VP & MG & F & F \\
\hline Community equity $\left(x_{2}^{3}\right)$ & Third priority & VP & G & MG & VG \\
\hline Historic impact $\left(x_{1}^{4}\right)$ & Fourth priority & VP & G & VP & P \\
\hline
\end{tabular}

In Step 3, the linguistic variables in Table 1 were used to describe the ratings of the candidate sites with respect to each criterion, as indicated in Table 3. After converting the linguistic terms to IT2TrF numbers, the IT2TrF rating $A_{i j}^{\kappa}$ was obtained for $z_{i} \in Z$ on $x_{j}^{\kappa} \in X^{\kappa}$. In Step 4, the synthesised value $Q_{i}^{\kappa}$ of alternative $z_{i} \in Z$ in each priority class was calculated. The computational results of $Q_{i}^{\kappa}$ are provided in Table 4 . Consider $Q_{2}^{1}(i=$ 2 and $\kappa=1$ ) as an example. As indicated in Table 3, the linguistic ratings of $z_{2}$ with respect to $x_{1}^{1}$ and $x_{2}^{1}$ are fair (F) and medium good (MG), respectively. According to Table 1, the corresponding IT2TrF numbers are $A_{21}^{1}=[(0.4025,0.4525,0.5375,0.5675 ; 0.8),(0.32,0.41$, $0.58,0.65 ; 1.0)]$ and $A_{22}^{1}=[(0.65,0.6725,0.7575,0.79 ; 0.8),(0.58,0.63,0.80,0.86 ; 1.0)]$. Next, applying (10), $Q_{2}^{1}$ is calculated as follows:

$$
\begin{aligned}
& Q_{2}^{1}=A_{21}^{1} \otimes A_{22}^{1}= \\
& {[(0.4025 \times 0.65,0.4525 \times 0.6725,0.5375 \times 0.7575,0.5675 \times 0.79 ; \min \{0.8,0.8\}),} \\
& (0.32 \times 0.58,0.41 \times 0.63,0.58 \times 0.80,0.65 \times 0.86 ; \min \{1.0,1.0\})]= \\
& {[(0.2616,0.3043,0.4072,0.4483 ; 0.8),(0.1856,0.2583,0.4640,0.5590 ; 1.0)] .}
\end{aligned}
$$


Table 4. Results for the synthesised values

\begin{tabular}{rrl}
\hline$z_{i}$ & $\kappa$ & The synthesised value $Q_{i}^{\kappa}$ \\
\hline$z_{1}$ & 0 & {$[(1.0000,1.0000,1.0000,1.0000 ; 1.0),(1.0000,1.0000,1.0000,1.0000 ; 1.0)]$} \\
\hline & 1 & {$[(0.6123,0.6642,0.7832,0.8236 ; 0.8),(0.5184,0.6084,0.8464,0.9409 ; 1.0)]$} \\
\hline & 2 & {$[(0.0875,0.1200,0.1600,0.1825 ; 0.8),(0.0400,0.1000,0.1800,0.2300 ; 1.0)]$} \\
\hline & 3 & {$[(0.0001,0.0001,0.0002,0.0028 ; 0.8),(0.0000,0.0000,0.0004,0.0049 ; 1.0)]$} \\
\hline$z_{2}$ & 0 & {$[(1.0000,1.0000,1.0000,1.0000 ; 1.0),(1.0000,1.0000,1.0000,1.0000 ; 1.0)]$} \\
\hline & 1 & {$[(0.2616,0.3043,0.4072,0.4483 ; 0.8),(0.1856,0.2583,0.4640,0.5590 ; 1.0)]$} \\
\hline & 2 & {$[(0.0071,0.0074,0.0149,0.0521 ; 0.8),(0.0000,0.0000,0.0200,0.0700 ; 1.0)]$} \\
\hline & 3 & {$[(0.5086,0.5481,0.6704,0.7169 ; 0.8),(0.4176,0.4914,0.7360,0.8342 ; 1.0)]$} \\
\hline$z_{3}$ & 0 & {$[(1.0000,1.0000,1.0000,1.0000 ; 1.0),(1.0000,1.0000,1.0000,1.0000 ; 1.0)]$} \\
\hline & 1 & {$[(0.0077,0.0144,0.0256,0.0333 ; 0.8),(0.0016,0.0100,0.0324,0.0529 ; 1.0)]$} \\
\hline & 2 & {$[(0.0075,0.0075,0.0150,0.0525 ; 0.8),(0.0000,0.0000,0.0200,0.0700 ; 1.0)]$} \\
\hline 3 & {$[(0.2616,0.3043,0.4072,0.4483 ; 0.8),(0.1856,0.2583,0.4640,0.5590 ; 1.0)]$} \\
\hline$z_{4}$ & 0 & {$[(1.0000,1.0000,1.0000,1.0000 ; 1.0),(1.0000,1.0000,1.0000,1.0000 ; 1.0)]$} \\
\hline & 1 & {$[(0.0203,0.0306,0.0520,0.0652 ; 0.8),(0.0068,0.0220,0.0648,0.0966 ; 1.0)]$} \\
\hline & 2 & {$[(0.0685,0.0978,0.1416,0.1656 ; 0.8),(0.0288,0.0780,0.1656,0.2231 ; 1.0)]$} \\
\hline & 3 & {$[(0.3814,0.4457,0.5335,0.5632 ; 0.8),(0.2976,0.4018,0.5800,0.6500 ; 1.0)]$} \\
\hline
\end{tabular}

In Step 5, the priority-based weight $W_{i}^{\kappa}$ of the $\kappa$ th priority class for each alternative $z_{i}$ $\in Z$ was computed. For example, $W_{2}^{3}$ is calculated as follows:

$$
\begin{aligned}
& W_{2}^{3}=Q_{2}^{0} \otimes Q_{2}^{1} \otimes Q_{2}^{2}= \\
& {[(1 \times 0.2616 \times 0.0071,1 \times 0.3043 \times 0.0074,1 \times 0.4072 \times 0.0149,1 \times 0.4483 \times 0.0521 ;} \\
& \min \{1.0,0.8,0.8\}),(1 \times 0.1856 \times 0.0000,1 \times 0.2583 \times 0.0000,1 \times 0.4640 \times 0.0200, \\
& 1 \times 0.5590 \times 0.0700 ; \min \{1.0,1.0,1.0\})]= \\
& {[(0.0019,0.0023,0.0061,0.0234 ; 0.8),(0.0000,0.0000,0.0093,0.0391 ; 1.0)] .}
\end{aligned}
$$

Next, the normalised priority-based weight $W_{i}^{\prime \kappa}$ for each $\kappa$ and $i$ was determined. For example, $W_{1}^{\prime 2}$ is calculated as follows:

$$
\begin{aligned}
& W_{1}^{\prime 2}=W_{1}^{2} \varnothing\left[\left(n_{1} \cdot W_{1}^{1}\right) \oplus\left(n_{2} \cdot W_{1}^{2}\right) \oplus\left(n_{3} \cdot W_{1}^{3}\right) \oplus\left(n_{4} \cdot W_{1}^{4}\right)\right]= \\
& {\left[\left(\frac{0.6123}{2 \times 1+2 \times 0.8236+2 \times 0.1503+1 \times 0.0004}, \frac{0.6642}{2 \times 1+2 \times 0.7832+2 \times 0.1253+1 \times 0},\right.\right.} \\
& \frac{0.7832}{2 \times 1+2 \times 0.6642+2 \times 0.0797+1 \times 0}, \frac{0.8236}{2 \times 1+2 \times 0.6123+2 \times 0.0536+1 \times 0} ; \\
& \min \{0.8, \min \{1.0,0.8,0.8,0.8\}\}),\left(\frac{0.5184}{2 \times 1+2 \times 0.9409+2 \times 0.2164+1 \times 0.0011},\right. \\
& \frac{0.6084}{2 \times 1+2 \times 0.8464+2 \times 0.1524+1 \times 0.0001}, \frac{0.8464}{2 \times 1+2 \times 0.6084+2 \times 0.0608+1 \times 0},
\end{aligned}
$$




$$
\begin{aligned}
& \left.\left.\frac{0.9409}{2 \times 1+2 \times 0.5184+2 \times 0.0207+1 \times 0} ; \min \{1.0, \min \{1.0,1.0,1.0,1.0\}\}\right)\right]= \\
& {[(0.1551,0.1740,0.2246,0.2472 ; 0.8),(0.1201,0.1522,0.2535,0.3057 ; 1.0)] .}
\end{aligned}
$$

The computational results of $W_{i}^{\kappa}$ and $W_{i}^{\prime \kappa}$ are provided in Table 5.

Table 5. Results for the (normalised) priority-based weights

\begin{tabular}{rrl}
\hline$z_{i}$ & $\kappa$ & The priority-based weight $W_{i}^{\kappa}$ \\
\hline$z_{1}$ & 1 & {$[(1.0000,1.0000,1.0000,1.0000 ; 1.0),(1.0000,1.0000,1.0000,1.0000 ; 1.0)]$} \\
\hline & 2 & {$[(0.6123,0.6642,0.7832,0.8236 ; 0.8),(0.5184,0.6084,0.8464,0.9409 ; 1.0)]$} \\
\hline & 3 & {$[(0.0536,0.0797,0.1253,0.1503 ; 0.8),(0.0207,0.0608,0.1524,0.2164 ; 1.0)]$} \\
\hline & 4 & {$[(0.0000,0.0000,0.0000,0.0004 ; 0.8),(0.0000,0.0000,0.0001,0.0011 ; 1.0)]$} \\
\hline$z_{2}$ & 1 & {$[(1.0000,1.0000,1.0000,1.0000 ; 1.0),(1.0000,1.0000,1.0000,1.0000 ; 1.0)]$} \\
\hline & 2 & {$[(0.2616,0.3043,0.4072,0.4483 ; 0.8),(0.1856,0.2583,0.4640,0.5590 ; 1.0)]$} \\
\hline & 3 & {$[(0.0019,0.0023,0.0061,0.0234 ; 0.8),(0.0000,0.0000,0.0093,0.0391 ; 1.0)]$} \\
\hline & 4 & {$[(0.0009,0.0012,0.0041,0.0167 ; 0.8),(0.0000,0.0000,0.0068,0.0326 ; 1.0)]$} \\
\hline$z_{3}$ & 1 & {$[(1.0000,1.0000,1.0000,1.0000 ; 1.0),(1.0000,1.0000,1.0000,1.0000 ; 1.0)]$} \\
\hline & 2 & {$[(0.0077,0.0144,0.0256,0.0333 ; 0.8),(0.0016,0.0100,0.0324,0.0529 ; 1.0)]$} \\
\hline & 3 & {$[(0.0001,0.0001,0.0004,0.0017 ; 0.8),(0.0000,0.0000,0.0006,0.0037 ; 1.0)]$} \\
\hline & 4 & {$[(0.0000,0.0000,0.0002,0.0008 ; 0.8),(0.0000,0.0000,0.0003,0.0021 ; 1.0)]$} \\
\hline$z_{4}$ & 1 & {$[(1.0000,1.0000,1.0000,1.0000 ; 1.0),(1.0000,1.0000,1.0000,1.0000 ; 1.0)]$} \\
\hline & 2 & {$[(0.0203,0.0306,0.0520,0.0652 ; 0.8),(0.0068,0.0220,0.0648,0.0966 ; 1.0)]$} \\
\hline & 3 & {$[(0.0014,0.0030,0.0074,0.0108 ; 0.8),(0.0002,0.0017,0.0107,0.0216 ; 1.0)]$} \\
\hline & 4 & {$[(0.0005,0.0013,0.0039,0.0061 ; 0.8),(0.0001,0.0007,0.0062,0.0140 ; 1.0)]$} \\
\hline$z_{i}$ & $\kappa$ & The normalised priority-based weight $W_{i}^{\prime \kappa}$ \\
\hline$z_{1}$ & 1 & {$[(0.2533,0.2620,0.2867,0.3001 ; 0.8),(0.2317,0.2501,0.2995,0.3249 ; 1.0)]$} \\
\hline & 2 & {$[(0.1551,0.1740,0.2246,0.2472 ; 0.8),(0.1201,0.1522,0.2535,0.3057 ; 1.0)]$} \\
\hline & 3 & {$[(0.4634,0.4711,0.4834,0.4893 ; 0.8),(0.4444,0.4636,0.4883,0.4965 ; 1.0)]$} \\
\hline & 3 & {$[(0.0136,0.0209,0.0359,0.0451 ; 0.8),(0.0048,0.0152,0.0457,0.0703 ; 1.0)]$} \\
\hline & 4 & {$[(0.0000,0.0000,0.0000,0.0001 ; 0.8),(0.0000,0.0000,0.0000,0.0004 ; 1.0)]$} \\
\hline$z_{2}$ & 1 & {$[(0.3378,0.3533,0.3825,0.3956 ; 0.8),(0.3097,0.3386,0.3974,0.4217 ; 1.0)]$} \\
\hline & 2 & {$[(0.0884,0.1075,0.1558,0.1773 ; 0.8),(0.0575,0.0875,0.1844,0.2357 ; 1.0)]$} \\
\hline & 3 & {$[(0.0006,0.0008,0.0023,0.0093 ; 0.8),(0.0000,0.0000,0.0037,0.0165 ; 1.0)]$} \\
\hline & & {$[(0.0003,0.0004,0.0016,0.0066 ; 0.8),(0.0000,0.0000,0.0027,0.0137 ; 1.0)]$} \\
\hline & & {$[(0.0037,0.0070,0.0126,0.0165 ; 0.8),(0.0008,0.0048,0.0160,0.0264 ; 1.0)]$} \\
\hline & & {$[(0.0000,0.0000,0.0002,0.0008 ; 0.8),(0.0000,0.0000,0.0003,0.0018 ; 1.0)]$} \\
\hline & & \\
\hline & &
\end{tabular}


In Step 6, the prioritised IT2F aggregation operator was employed to acquire the synthetic evaluation of alternative $z_{i} \in Z$. Consider alternative $z_{1}$ as an example. According to (19),

$\operatorname{PIT} 2 F A\left(A_{11}, A_{12}, \cdots, A_{17}\right)=\left(W_{1}^{\prime 1} \otimes A_{11}^{1}\right) \oplus\left(W_{1}^{\prime 1} \otimes A_{12}^{1}\right) \oplus\left(W_{1}^{\prime 2} \otimes A_{11}^{2}\right) \oplus\left(W_{1}^{\prime 2} \otimes A_{12}^{2}\right)=$ $\oplus\left(W_{1}^{\prime 3} \otimes A_{11}^{3}\right) \oplus\left(W_{1}^{\prime 3} \otimes A_{12}^{3}\right) \oplus\left(W_{1}^{\prime 4} \otimes A_{11}^{4}\right)$.

Next, the PIT2FA value of $z_{1}$ was calculated as follows:

$\operatorname{PIT} 2 F A\left(A_{11}, A_{12}, \cdots, A_{17}\right)=[(0.5653,0.6223,0.7691,0.8417 ; 0.8),(0.4586,0.5576$, $0.8520,1.0162 ; 1.0)]$,

where, e.g.,

$$
\begin{aligned}
& \sum_{\kappa=1}^{4} \sum_{j=1}^{n_{\kappa}} w_{11}^{\prime \kappa L} \cdot a_{11 j}^{\kappa L}=0.2533 \times 0.7825+0.2533 \times 0.7825+0.1551 \times 1+0.1551 \times 0.0875+ \\
& 0.0136 \times 0.0075+0.0136 \times 0.0075+0.0000 \times 0.0075=0.5653 .
\end{aligned}
$$

The PIT2FA values of the other three alternatives are as follows:

$\operatorname{PIT} 2 F A\left(A_{21}, A_{22}, \cdots, A_{27}\right)=[(0.4411,0.5057,0.6575,0.7441 ; 0.8),(0.3322,0.4379$, $0.7453,0.9325 ; 1.0)]$,

$\operatorname{PIT} 2 F A\left(A_{31}, A_{32}, \cdots, A_{37}\right)=[(0.0882,0.1240,0.1708,0.1995 ; 0.8),(0.0386,0.1016$, $0.1949,0.2607 ; 1.0)]$,

$\operatorname{PIT} 2 F A\left(A_{41}, A_{42}, \cdots, A_{47}\right)=[(0.1573,0.1922,0.2665,0.3078 ; 0.8),(0.0957,0.1585$, $0.3072,0.3996 ; 1.0)]$.

In Step 7, the signed distance for each alternative $z_{i} \in Z$ was computed using (22), as follows:

$$
\begin{aligned}
& d\left(\operatorname{PIT} 2 F A\left(A_{11}, A_{12}, \cdots, A_{1 n}\right), \tilde{0}_{1}\right)= \\
& \frac{1}{8}[0.5653+0.6223+0.7691+0.8417+4 \times 0.4586+2 \times 0.5576+2 \times 0.8520+ \\
& \left.4 \times 1.0162+3(0.5576+0.8520-0.4586-1.0162) \frac{0.8}{1.0}\right]=1.4200
\end{aligned}
$$

$d\left(\operatorname{PIT} 2 F A\left(A_{21}, A_{22}, \cdots, A_{2 n}\right), \tilde{0}_{1}\right)=1.1973, \quad d\left(\operatorname{PIT} 2 F A\left(A_{31}, A_{32}, \cdots, A_{3 n}\right), \tilde{0}_{1}\right)=0.2957$, and $d\left(\operatorname{PIT} 2 F A\left(A_{41}, A_{42}, \cdots, A_{4 n}\right), \tilde{0}_{1}\right)=0.4707$. Next, the complete ranking orders of the four

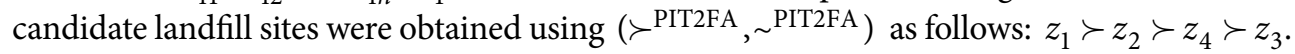
The best choice is the first candidate site $\left(z_{1}\right)$.

\section{Conclusions}

Exact data can be difficult to determine precisely because human judgment is often imprecise under many conditions. At times, available information is not sufficient for an exact definition of a degree of membership for certain elements. The use of IT2F sets can appropriately address imprecise or uncertain decision information in fields that require MCDA, especially with respect to a lack of knowledge or experience, intangible or nonmonetary criteria, or a complex and uncertain environment. In the decision context of 
IT2 TrF numbers, this paper developed a prioritised IT2F aggregation-operator-based approach to address MCDA problems in which prioritisation relationships exist among the evaluative criteria. This paper modelled prioritisation among the criteria by assessing the priority-based weights that are associated with criteria dependence on the satisfaction of the higher priority criteria. This paper presented a new prioritised IT2F aggregation operator to aggregate the IT2 $\mathrm{TrF}$ ratings of alternatives with respect to each prioritised criterion. Based on synthetic evaluations given by the prioritised IT2F aggregation operator, this paper determined the ranking order of the alternatives according to the corresponding signed distances. Furthermore, this paper explored the problem of landfill site selection to demonstrate the feasibility and applicability of the proposed method.

\section{Acknowledgments}

The author is very grateful to the respected editor and the anonymous referees for their insightful and constructive comments, which helped to improve the overall quality of the paper. The author is grateful to the grant funding support from the Taiwan Ministry of Science and Technology (MOST 105-2410-H-182-007-MY3) and Chang Gung Memorial Hospital (BMRP 574) during which the study was completed.

\section{References}

Baležentis, T.; Zeng, S. 2013. Group multi-criteria decision making based upon interval-valued fuzzy numbers: an extension of the MULTIMOORA method, Expert Systems with Applications 40(2): 543-550. https://doi.org/10.1016/j.eswa.2012.07.066

Chen, T.-Y. 2011. Signed distanced-based TOPSIS method for multiple criteria decision analysis based on generalized interval-valued fuzzy numbers, International Journal of Information Technology \& Decision Making 10(6): 1131-1159. https://doi.org/10.1142/S0219622011004749

Chen, T.-Y. 2012. Multiple criteria group decision-making with generalized interval-valued fuzzy numbers based on signed distances and incomplete weights, Applied Mathematical Modelling 36(7): 3029-3052. https://doi.org/10.1016/j.apm.2011.09.080

Chen, T.-Y.; Chang, C.-H.; Lu, J.-f. R. 2013. The extended QUALIFLEX method for multiple criteria decision analysis based on interval type-2 fuzzy sets and applications to medical decision making, European Journal of Operational Research 226(3): 615-625. https://doi.org/10.1016/j.ejor.2012.11.038

Chen, S.-M.; Lee, L.-W. 2010. Fuzzy multiple attributes group decision-making based on the ranking values and the arithmetic operations of interval type-2 fuzzy sets, Expert Systems with Applications 37(1): 824-833. https://doi.org/10.1016/j.eswa.2009.06.094

Gilan, S. S.; Sebt, M. H.; Shahhosseini, V. 2012. Computing with words for hierarchical competency based selection of personnel in construction companies, Applied Soft Computing 12(2): 860-871. https://doi.org/10.1016/j.asoc.2011.10.004

Greenfield, S.; Chiclana, F.; Coupland, S.; John, R. 2009. The collapsing method of defuzzification for discretised interval type-2 fuzzy sets, Information Sciences 179(13): 2055-2069.

https://doi.org/10.1016/j.ins.2008.07.011

Han, Z.; Liu, P. 2011. A fuzzy multi-attribute decision-making method under risk with unknown attribute weights, Technological and Economic Development of Economy 17(2): 246-258.

https://doi.org/10.3846/20294913.2011.580575 
Lai, H.-L.; Chen, T.-Y. 2015. Client acceptance method for audit firms based on interval-valued fuzzy numbers, Technological and Economic Development of Economy 21(1): 1-27. https://doi.org/10.3846/20294913.2014.871662

Li, B.; He, W. 2012. Intuitionistic fuzzy PRI-AND and PRI-OR aggregation operators, Information Fusion. https://doi.org/10.1016/j.inffus.2012.10.006

Liou, J. J. H.; Tzeng, G. H. 2012. Comments on "multiple criteria decision making (MCDM) methods in economics: an overview", Technological and Economic Development of Economy 18(4): 672-695. https://doi.org/10.3846/20294913.2012.753489

Mendel, J. M. 2007. Type-2 fuzzy sets and systems: an overview, IEEE Computational Intelligence Magazine 2(1): 20-29. https://doi.org/10.1109/MCI.2007.380672

Mendel, J. M.; John, R. I.; Liu, F. 2006. Interval type-2 fuzzy logic systems made simple, IEEE Transactions on Fuzzy Systems 14(6): 808-821. https://doi.org/10.1109/TFUZZ.2006.879986

Rajpathak, D.; Chougule, R.; Bandyopadhyay, P. 2012. A domain-specific decision support system for knowledge discovery using association and text mining, Knowledge and Information Systems 31(3): 405-432. https://doi.org/10.1007/s10115-011-0409-1

Stanujkic, D.; Magdalinovic, N.; Jovanovic, R.; Stojanovic, S. 2012. An objective multi-criteria approach to optimization using MOORA method and interval grey numbers, Technological and Economic Development of Economy 18(2): 331-363. https://doi.org/10.3846/20294913.2012.676996

Wang, W.; Liu, X.; Qin, Y. 2012. Multi-attribute group decision making models under interval type-2 fuzzy environment, Knowledge-Based Systems 30: 121-128. https://doi.org/10.1016/j.knosys.2012.01.005

Wei, G. 2012. Hesitant fuzzy prioritized operators and their application to multiple attribute decision making, Knowledge-Based Systems 31: 176-182. https://doi.org/10.1016/j.knosys.2012.03.011

Wei, G.; Zhao, X.; Wang, H. 2012. An approach to multiple attribute group decision making with interval intuitionistic trapezoidal fuzzy information, Technological and Economic Development of Economy 18(2): 317-330. https://doi.org/10.3846/20294913.2012.676995

Xu, Y.-J.; Sun, T.; Li, D.-F. 2011. Intuitionistic fuzzy prioritized OWA operator and its application in multi-criteria decision-making problem, Kongzhi yu Juece/Control and Decision 26(1): 129-132.

Yager, R. R. 2004. Modeling prioritized multi-criteria decision making, IEEE Transactions on Systems, Man and Cybernetics, Part B. Cybernetics 34(6): 2396-2404.

https://doi.org/10.1109/TSMCB.2004.837348

Yager, R. R. 2008. Prioritized aggregation operators, International Journal of Approximate Reasoning 48(1): 263-274. https://doi.org/10.1016/j.ijar.2007.08.009

Yager, R. R. 2009. Prioritized OWA aggregation, Fuzzy Optimization and Decision Making 8(3): 245262. https://doi.org/10.1007/s10700-009-9063-4

Yager, R. R.; Giray, G.; Marek, Z. 2011. Using a web personal evaluation tool-PET for lexicographic multi-criteria service selection, Knowledge-Based Systems 24(7): 929-942. https://doi.org/10.1016/j.knosys.2011.02.004

Yu, D. 2012. Group decision making based on generalized intuitionistic fuzzy prioritized geometric operator, International Journal of Intelligent Systems 27(7): 635-661. https://doi.org/10.1002/int.21538

Yu, D.; Wu, Y.; Lu, T. 2012. Interval-valued intuitionistic fuzzy prioritized operators and their application in group decision making, Knowledge-Based Systems 30: 57-66. https://doi.org/10.1016/j.knosys.2011.11.004

Yu, X.; Xu, Z. 2013. Prioritized intuitionistic fuzzy aggregation operators, Information Fusion 14(1): 108-116. https://doi.org/10.1016/j.inffus.2012.01.011

Zadeh, L. A. 1975. The concept of a linguistic variable and its application to approximate reasoning-I, Information Sciences 8(3): 199-249. https://doi.org/10.1016/0020-0255(75)90036-5 
Zavadskas, E. K.; Turskis, Z. 2011. Multiple criteria decision making (MCDM) methods in economics: an overview, Technological and Economic Development of Economy 17(2): 397-427. https://doi.org/10.3846/20294913.2011.593291

Zhai, D.; Mendel, J. M. 2011. Uncertainty measures for general Type-2 fuzzy sets, Information Sciences 181(3): 503-518. https://doi.org/10.1016/j.ins.2010.09.020

Zhang, Z.; Zhang, S. 2013. A novel approach to multi attribute group decision making based on trapezoidal interval type-2 fuzzy soft sets, Applied Mathematical Modelling 37(7): 4948-4971. https://doi.org/10.1016/j.apm.2012.10.006

Zhao, X.; Lin, R.; Wei, G. 2013. Fuzzy prioritized operators and their application to multiple attribute group decision making, Applied Mathematical Modelling 37(7): 4759-4770.

https://doi.org/10.1016/j.apm.2012.09.048

\section{Appendix A}

Proof of Theorem 1. Because $A_{i j}^{\kappa}$ is an IT2TrF number bounded within [0,1], $0 \leq a_{1 i j}^{\kappa L} \leq$ $a_{2 i j}^{\kappa L} \leq a_{3 i j}^{\kappa L} \leq a_{4 i j}^{\kappa L} \leq 1,0 \leq a_{1 i j}^{\kappa U} \leq a_{2 i j}^{\kappa U} \leq a_{3 i j}^{\kappa U} \leq a_{4 i j}^{\kappa U} \leq 1, a_{1 i j}^{\kappa U} \leq a_{1 i j}^{\kappa L}, a_{4 i j}^{\kappa L} \leq a_{4 i j}^{\kappa U}, 0<h_{i j}^{\kappa L} \leq h_{i j}^{\kappa U} \leq 1$, and $A_{i j}^{\kappa L} \subseteq A_{i j}^{\kappa U}$ hold for each $i=1,2, \cdots, m, j=1,2, \cdots, n_{\kappa}$, and $\kappa=1,2, \cdots, \eta$. It follows that $0 \leq \prod_{j=1}^{n_{\kappa}} a_{1 i j}^{\kappa L} \leq \prod_{j=1}^{n_{\kappa}} a_{2 i j}^{\kappa L} \leq \prod_{j=1}^{n_{\kappa}} a_{3 i j}^{\kappa L} \leq \prod_{j=1}^{n_{\kappa}} a_{4 i j}^{\kappa L} \leq 1, \quad 0 \leq \prod_{j=1}^{n_{\kappa}} a_{1 i j}^{\kappa U} \leq \prod_{j=1}^{n_{\kappa}} a_{2 i j}^{\kappa U} \leq$ $\prod_{j=1}^{n_{\kappa}} a_{3 i j}^{\kappa U} \leq \prod_{j=1}^{n_{\kappa}} a_{4 i j}^{\kappa U} \leq 1, \quad \prod_{j=1}^{n_{\kappa}} a_{1 i j}^{\kappa U} \leq \prod_{j=1}^{n_{\kappa}} a_{1 i j}^{\kappa L}, \quad \prod_{j=1}^{n_{\kappa}} a_{4 i j}^{\kappa L} \leq \prod_{j=1}^{n_{\kappa}} a_{4 i j}^{\kappa U}, \quad$ and $0<\min _{j=1}^{n_{\kappa}} h_{i j}^{\kappa L} \leq \min _{j=1}^{n_{\kappa}} h_{i j}^{\kappa U} \leq 1$. By Definition 5 and (13), $0 \leq q_{1 i}^{\kappa L} \leq q_{2 i}^{\kappa L} \leq q_{3 i}^{\kappa L} \leq q_{4 i}^{\kappa L} \leq 1$, $0 \leq q_{1 i}^{\kappa U} \leq q_{2 i}^{\kappa U} \leq q_{3 i}^{\kappa U} \leq q_{4 i}^{\kappa U} \leq 1, q_{1 i}^{\kappa U} \leq q_{1 i}^{\kappa L}, q_{4 i}^{\kappa L} \leq q_{4 i}^{\kappa U}, 0<h_{q i}^{\kappa L} \leq h_{q i}^{\kappa U} \leq 1$, and $Q_{i}^{\kappa L} \subseteq Q_{i}^{\kappa U}$ for each $\kappa=0,1, \cdots, \eta-1$ and $i=1,2, \cdots, m$. Therefore, the synthesised value $Q_{i}^{\kappa}$ is an IT$2 \mathrm{TrF}$ number bounded within $[0,1]$. The proof of the priority-based weight $W_{i}^{\mathrm{K}}$ is similar to that of $Q_{i}^{\kappa}$.

\section{Appendix B}

Proof of Theorem 2. Let $\xi \in\{1,2,3,4\}$. The following inequalities hold for each $\xi$, as follows:

$$
\prod_{\varphi=1}^{\kappa} q_{\xi i}^{\varphi-1, L} \geq \prod_{\varphi=1}^{\kappa} q_{\xi i}^{\varphi-1, L} \cdot \prod_{\varphi=\kappa+1}^{\kappa "} q_{\xi i}^{\varphi-1, L} \text { and } \prod_{\varphi=1}^{\kappa} q_{\xi i}^{\varphi-1, U} \geq \prod_{\varphi=1}^{\kappa} q_{\xi i}^{\varphi-1, U} \cdot \prod_{\varphi=\kappa+1}^{\kappa "} q_{\xi i}^{\varphi-1, U} .
$$

Therefore, $w_{\xi_{i}}^{\kappa L} \geq w_{\xi_{i}}^{\kappa " L}$ and $w_{\xi i}^{\kappa U} \geq w_{\xi_{i}}^{\kappa " U}$ for each $\xi$. In addition,

$$
\begin{aligned}
& \min _{\varphi=1}^{\kappa} h_{q i}^{\varphi-1, L} \geq \min \left\{\min _{\varphi=1}^{\kappa} h_{q i}^{\varphi-1, L}, \min _{\varphi=\kappa+1}^{\kappa "} h_{q i}^{\varphi-1, L}\right\}=\min _{\varphi=1}^{\kappa "} h_{q i}^{\varphi-1, L} \text { and } \\
& \min _{\varphi=1}^{\kappa} h_{q i}^{\varphi-1, U} \geq \min \left\{\min _{\varphi=1}^{\kappa} h_{q i}^{\varphi-1, U}, \underset{\varphi=\kappa+1}{\min ^{\prime \prime}} h_{q i}^{\varphi-1, U}\right\}=\min _{\varphi=1}^{\kappa "} h_{q i}^{\varphi-1, U} .
\end{aligned}
$$

In other words, $h_{w i}^{\kappa L} \geq h_{w i}^{\kappa "} L$ and $h_{w i}^{\kappa U} \geq h_{w i}^{\kappa "} U$ hold. It follows that $W_{i}^{\mathrm{\kappa}} \supseteq W_{i}^{\kappa "}$. 


\section{Appendix C}

Proof of Theorem 3. Because $W_{i}^{\kappa}$ is an IT2TrF number bounded within $[0,1], 0 \leq w_{1 i}^{\kappa L}$ $\leq w_{2 i}^{\kappa L} \leq w_{3 i}^{\kappa L} \leq w_{4 i}^{\kappa L} \leq 1, \quad 0 \leq w_{1 i}^{\kappa U} \leq w_{2 i}^{\kappa U} \leq w_{3 i}^{\kappa U} \leq w_{4 i}^{\kappa U} \leq 1, \quad w_{1 i}^{\kappa U} \leq w_{1 i}^{\kappa L}, \quad w_{4 i}^{\kappa L} \leq w_{4 i}^{\kappa U}$, $0<h_{w i}^{\kappa L} \leq h_{w i}^{\kappa U} \leq 1$, and $W_{i}^{\kappa L} \subset W_{i}^{\kappa U}$ hold for each $i=1,2, \cdots, m$ and $\kappa=1,2, \cdots, \eta$. Consider the case of $\kappa=1$. According to (13), it is known that $W_{i}^{1}=\left[\left(q_{1 i}^{0, L}, q_{2 i}^{0, L}, q_{3 i}^{0, L}, q_{4 i}^{0, L} ; h_{q i}^{0, L}\right)\right.$, $\left.\left(q_{1 i}^{0, U}, q_{2 i}^{0, U}, q_{3 i}^{0, U}, q_{4 i}^{0, U} ; h_{q i}^{0, U}\right)\right]$ for $i=1,2, \cdots, m$. By $(11)$, it follows that $W_{i}^{1}=[(1,1,1,1 ; 1)$, $(1,1,1,1 ; 1)]$ for $i=1,2, \cdots, m$. Recall that $n_{\kappa}$ denotes the number of criteria in $X^{\kappa}$; thus, $n_{\kappa} \geq 1$ must hold for each $\kappa=1,2, \cdots, \eta$. Let $\xi \in\{1,2,3,4\}$. The following inequality is satisfied for each $\xi$ :

$\sum_{\gamma=1}^{\eta} n_{\gamma} \cdot w_{\xi i}^{\gamma L}=n_{1} \cdot w_{\xi i}^{1 L}+\sum_{\gamma=2}^{\eta} n_{\gamma} \cdot w_{\xi i}^{\gamma L}=n_{1} \cdot 1+\sum_{\gamma=2}^{\eta} n_{\gamma} \cdot w_{\xi i}^{\gamma L} \geq n_{1} \geq 1$

Because $0 \leq w_{\xi i}^{\kappa L} \leq 1$ and $0 \leq w_{\xi i}^{\kappa U} \leq 1$ for each $\xi, 0 \leq w_{\xi i}^{\kappa L} / \sum_{\gamma=1}^{\eta} n_{\gamma} \cdot w_{(5-\xi) i}^{\gamma L} \leq 1$.

However, because $w_{1 i}^{\kappa L} \leq w_{2 i}^{\kappa L}$ and $\sum_{\gamma=1}^{\eta} n_{\gamma} \cdot w_{4 i}^{\gamma L} \geq \sum_{\gamma=1}^{\eta} n_{\gamma} \cdot w_{3 i}^{\gamma L}$, it follows that $w_{1 i}^{\kappa L} / \sum_{\gamma=1}^{\eta} n_{\gamma} \cdot w_{4 i}^{\gamma L} \geq w_{2 i}^{\kappa L} / \sum_{\gamma=1}^{\eta} n_{\gamma} \cdot w_{3 i}^{\gamma L}$. Similarly, it is known that

$$
\begin{aligned}
& \frac{w_{1 i}^{\kappa L}}{\sum_{\gamma=1}^{\eta} n_{\gamma} \cdot w_{4 i}^{\gamma L}} \leq \frac{w_{2 i}^{\kappa L}}{\sum_{\gamma=1}^{\eta} n_{\gamma} \cdot w_{3 i}^{\gamma L}} \leq \frac{w_{3 i}^{\kappa L}}{\sum_{\gamma=1}^{\eta} n_{\gamma} \cdot w_{2 i}^{\gamma L}} \leq \frac{w_{4 i}^{\kappa L}}{\sum_{\gamma=1}^{\eta} n_{\gamma} \cdot w_{1 i}^{\gamma L}} \text { and } \\
& \frac{w_{1 i}^{\kappa U}}{\sum_{\gamma=1}^{\eta} n_{\gamma} \cdot w_{4 i}^{\gamma U}} \leq \frac{w_{2 i}^{\kappa U}}{\sum_{\gamma=1}^{\eta} n_{\gamma} \cdot w_{3 i}^{\gamma U}} \leq \frac{w_{3 i}^{\kappa U}}{\sum_{\gamma=1}^{\eta} n_{\gamma} \cdot w_{2 i}^{\gamma U}} \leq \frac{w_{4 i}^{\kappa U}}{\sum_{\gamma=1}^{\eta} n_{\gamma} \cdot w_{1 i}^{\gamma U}} .
\end{aligned}
$$

According to the conditions of $w_{4 i}^{\kappa L} \leq w_{4 i}^{\kappa U}$ and $w_{1 i}^{\kappa L} \geq w_{1 i}^{\kappa U}, \sum_{\gamma=1}^{\eta} n_{\gamma} \cdot w_{4 i}^{\gamma L} \leq \sum_{\gamma=1}^{\eta} n_{\gamma} \cdot w_{4 i}^{\gamma U}$ and $\sum_{\gamma=1}^{\eta} n_{\gamma} \cdot w_{1 i}^{\gamma L} \geq \sum_{\gamma=1}^{\eta} n_{\gamma} \cdot w_{1 i}^{\gamma U}$ hold. Therefore, the following two inequalities are satisfied:

$$
\frac{w_{1 i}^{\kappa L}}{\sum_{\gamma=1}^{\eta} n_{\gamma} \cdot w_{4 i}^{\gamma L}} \geq \frac{w_{1 i}^{\kappa U}}{\sum_{\gamma=1}^{\eta} n_{\gamma} \cdot w_{4 i}^{\gamma U}} \text { and } j=1,2, \cdots, n_{\kappa} \text {. }
$$

By the condition of $0<h_{w i}^{\kappa L} \leq h_{w i}^{\kappa U} \leq 1$, it is obvious that $0<\min _{\gamma=1}^{\eta} h_{w i}^{\gamma L} \leq \min _{\gamma=1}^{\eta} h_{w i}^{\gamma U} \leq 1$. In addition, this condition implies that:

$$
0<\min \left\{h_{w i}^{\kappa L}, \min _{\gamma=1}^{\eta} h_{w i}^{\gamma L}\right\} \leq \min \left\{h_{w i}^{\kappa U}, \min _{\gamma=1}^{\eta} h_{w i}^{\gamma U}\right\} \leq 1 .
$$

Thus, $0 \leq w_{1 i}^{\prime \kappa L} \leq w_{2 i}^{\prime \kappa L} \leq w_{3 i}^{\prime \kappa L} \leq w_{4 i}^{\prime \kappa L} \leq 1,0 \leq w_{1 i}^{\prime \kappa U} \leq w_{2 i}^{\prime \kappa U} \leq w_{3 i}^{\prime \kappa U} \leq w_{4 i}^{\prime \kappa U} \leq 1, w_{1 i}^{\prime \kappa U} \leq w_{1 i}^{\prime \kappa L}$, $w_{4 i}^{\prime \kappa L} \leq w_{4 i}^{\prime \kappa U}, 0<h_{w^{\prime} i}^{\kappa L} \leq h_{w^{\prime} i}^{\kappa U} \leq 1$, and $W_{i}^{\prime \kappa L} \subset W_{i}^{\prime \kappa U}$ can be obtained for each $\kappa=1,2, \cdots, \eta$ and $i=1,2, \cdots, m$. Therefore, the normalised value $W_{i}^{\prime \kappa}$ is an IT2TrF number bounded within $[0,1]$. 


\section{Appendix D}

Proof of Theorem 5. Because $A_{i j}^{\kappa}$ is an IT2TrF number bounded within [0,1], $0 \leq a_{1 i j}^{\kappa L} \leq$ $a_{2 i j}^{\kappa L} \leq a_{3 i j}^{\kappa L} \leq a_{4 i j}^{\kappa L} \leq 1, \quad 0 \leq a_{1 i j}^{\kappa U} \leq a_{2 i j}^{\kappa U} \leq a_{3 i j}^{\kappa U} \leq a_{4 i j}^{\kappa U} \leq 1, \quad a_{1 i j}^{\kappa U} \leq a_{1 i j}^{\kappa L}, \quad a_{4 i j}^{\kappa L} \leq a_{4 i j}^{\kappa U}, \quad 0<h_{i j}^{\kappa L} \leq$ $h_{i j}^{\kappa U} \leq 1$, and $A_{i j}^{\kappa L} \subseteq A_{i j}^{\kappa U}$ hold for each $\kappa=1,2, \cdots, \eta, j=1,2, \cdots, n_{\kappa}$, and $i=1,2, \cdots, m$. Similarly, $0 \leq w_{1 i}^{\prime \kappa L} \leq w_{2 i}^{\prime \kappa L} \leq w_{3 i}^{\prime \kappa L} \leq w_{4 i}^{\prime \kappa L} \leq 1, \quad 0 \leq w_{1 i}^{\prime \kappa U} \leq w_{2 i}^{\prime \kappa U} \leq w_{3 i}^{\prime \kappa U} \leq w_{4 i}^{\prime \kappa U} \leq 1, \quad w_{1 i}^{\prime \kappa U} \leq$ $w_{1 i}^{\prime \kappa L}, w_{4 i}^{\prime \kappa L} \leq w_{4 i}^{\prime \kappa U}, 0<h_{w^{\prime} i}^{\kappa L} \leq h_{w^{\prime} i}^{\kappa U} \leq 1$, and $W_{i}^{\prime \kappa L} \subset W_{i}^{\prime \kappa U}$ hold for each $\kappa=1,2, \cdots, \eta$ and $i=1,2, \cdots, m$. According to $0 \leq a_{1 i j}^{\kappa L} \leq a_{2 i j}^{\kappa L} \leq a_{3 i j}^{\kappa L} \leq a_{4 i j}^{\kappa L} \leq 1, \quad 0 \leq a_{1 i j}^{\kappa U} \leq a_{2 i j}^{\kappa U} \leq a_{3 i j}^{\kappa U} \leq a_{4 i j}^{\kappa U} \leq 1$, $0 \leq w_{1 i}^{\prime \kappa L} \leq w_{2 i}^{\prime \kappa L} \leq w_{3 i}^{\prime \kappa L} \leq w_{4 i}^{\prime \kappa L} \leq 1$, and $0 \leq w_{1 i}^{\prime \kappa U} \leq w_{2 i}^{\prime \kappa U} \leq w_{3 i}^{\prime \kappa U} \leq w_{4 i}^{\prime \kappa U} \leq 1$, it is obvious that:

$$
\begin{aligned}
& 0 \leq \sum_{\kappa=1}^{\eta} \sum_{j=1}^{n_{\kappa}} w_{1 i}^{\prime \kappa L} \cdot a_{1 i j}^{\kappa L} \leq \sum_{\kappa=1}^{\eta} \sum_{j=1}^{n_{\kappa}} w_{2 i}^{\prime \kappa L} \cdot a_{2 i j}^{\kappa L} \leq \sum_{\kappa=1}^{\eta} \sum_{j=1}^{n_{\kappa}} w_{3 i}^{\prime \kappa L} \cdot a_{3 i j}^{\kappa L} \leq \sum_{\kappa=1}^{\eta} \sum_{j=1}^{n_{\kappa}} w_{4 i}^{\prime \kappa L} \cdot a_{4 i j}^{\kappa L} \text { and } \\
& 0 \leq \sum_{\kappa=1}^{\eta} \sum_{j=1}^{n_{\kappa}} w_{1 i}^{\prime \kappa U} \cdot a_{1 i j}^{\kappa U} \leq \sum_{\kappa=1}^{\eta} \sum_{j=1}^{n_{\kappa}} w_{2 i}^{\prime \kappa U} \cdot a_{2 i j}^{\kappa U} \leq \sum_{\kappa=1}^{\eta} \sum_{j=1}^{n_{\kappa}} w_{3 i}^{\prime \kappa U} \cdot a_{3 i j}^{\kappa U} \leq \sum_{\kappa=1}^{\eta} \sum_{j=1}^{n_{\kappa}} w_{4 i}^{\prime \kappa U} \cdot a_{4 i j}^{\kappa U} .
\end{aligned}
$$

Note that $\sum_{\kappa=1}^{\eta} \sum_{j=1}^{n_{\kappa}} w_{\xi i}^{\prime \kappa L} \cdot a_{\xi i j}^{\kappa L}$ and $\sum_{\kappa=1}^{\eta} \sum_{j=1}^{n_{\kappa}} w_{\xi i}^{\prime \kappa U} \cdot a_{\xi i j}^{\kappa U}$ might be larger than 1 for certain $\xi \in\{1,2,3,4\}$ because the sum of $W_{i}^{\prime \kappa}$ is not equal to $[(1,1,1,1 ; 1),(1,1,1,1 ; 1)]$. Because $a_{1 i j}^{\kappa U} \leq a_{1 i j}^{\kappa L}, a_{4 i j}^{\kappa L} \leq a_{4 i j}^{\kappa U}, w_{1 i}^{\prime \kappa U} \leq w_{1 i}^{\prime \kappa L}$, and $w_{4 i}^{\prime \kappa L} \leq w_{4 i}^{\prime \kappa U}$, it follows that:

$$
\sum_{\kappa=1}^{\eta} \sum_{j=1}^{n_{\kappa}} w_{1 i}^{\prime \kappa U} \cdot a_{1 i j}^{\kappa U} \leq \sum_{\kappa=1}^{\eta} \sum_{j=1}^{n_{\kappa}} w_{1 i}^{\prime \kappa L} \cdot a_{1 i j}^{\kappa L} \text { and } \sum_{\kappa=1}^{\eta} \sum_{j=1}^{n_{\kappa}} w_{4 i}^{\prime \kappa L} \cdot a_{4 i j}^{\kappa L} \leq \sum_{\kappa=1}^{\eta} \sum_{j=1}^{n_{\kappa}} w_{4 i}^{\prime \kappa U} \cdot a_{4 i j}^{\kappa U} .
$$

Finally, the conditions of $0<h_{i j}^{\kappa L} \leq h_{i j}^{\kappa U} \leq 1$ and $0<h_{w^{\prime} i}^{\kappa L} \leq h_{w^{\prime} i}^{\kappa U} \leq 1$ imply that:

$$
0<\min _{\kappa=1}^{\eta} \min _{j=1}^{n_{\kappa}}\left(\min \left\{h_{w^{\prime} i}^{\kappa L}, h_{i j}^{\kappa L}\right\}\right) \leq \min _{\kappa=1}^{\eta} \min _{j=1}^{n_{\kappa}}\left(\min \left\{h_{w^{\prime} i}^{\kappa U}, h_{i j}^{\kappa U}\right\}\right) \leq 1 \text {. }
$$

Therefore, $\operatorname{PIT} 2 F A\left(A_{i 1}, A_{i 2}, \cdots, A_{i n}\right)$ is an IT2TrF number.

\section{Appendix E}

Proof of Theorem 6. Let $\xi \in\{1,2,3,4\}$. For each $\xi$, because $A_{i j}^{\kappa} \subseteq A_{i j}^{\text {"k }}$ for all $\kappa, i$, and $j$, it is known that $a_{\xi i j}^{\kappa L} \leq a_{\xi i j}^{\mathrm{\kappa L}}, a_{\xi i j}^{\kappa U} \leq a_{\xi i j}^{\prime \kappa U}, h_{i j}^{\kappa L} \leq h_{i j}^{\prime \prime \kappa L}$, and $h_{i j}^{\kappa U} \leq h_{i j}^{\prime \kappa U}$. It follows that $\sum_{\kappa=1}^{\eta} \sum_{j=1}^{n_{\kappa}} w_{\xi i}^{\prime \kappa L} \cdot a_{\xi i j}^{\kappa L} \leq \sum_{\kappa=1}^{\eta} \sum_{j=1}^{n_{\kappa}} w_{\xi i}^{\prime \kappa L} \cdot a_{\xi i j}^{\prime \prime \kappa L}, \sum_{\kappa=1}^{\eta} \sum_{j=1}^{n_{\kappa}} w_{\xi i}^{\prime \kappa U} \cdot a_{\xi i j}^{\kappa U} \leq \sum_{\kappa=1}^{\eta} \sum_{j=1}^{n_{\kappa}} w_{\xi i}^{\prime \kappa U} \cdot a_{\xi i j}^{\prime \prime \kappa U}, \min _{\substack{\kappa=1 \\ \eta}}^{\eta} \min _{\substack{\kappa \\ n_{\kappa}}}^{n_{\kappa}}\left(\min \left\{h_{w^{\prime} i}^{\kappa L}\right.\right.$, $\left.\left.h_{i j}^{\kappa L}\right\}\right) \leq \min _{\kappa=1}^{\eta} \min _{j=1}^{n_{\kappa}}\left(\min \left\{h_{w^{\prime} i}^{\kappa L}, h_{i j}^{\prime \kappa L}\right\}\right)$, and $\min _{\kappa=1} \min _{j=1}\left(\min \left\{h_{w^{\prime} i}^{\kappa U}, h_{i j}^{\kappa U}\right\}\right) \leq \min _{\kappa=1} \min _{j=1}^{n_{\kappa}}\left(\min \left\{h_{w^{\prime} i}^{\kappa U}\right.\right.$, $\left.\left.h_{i j}^{\prime \kappa U}\right\}\right)$. Therefore, $\operatorname{PIT} 2 F A\left(A_{i 1}, A_{i 2}, \cdots, A_{i n}\right) \subseteq \operatorname{PIT} 2 F A\left(A_{i 1}^{\prime \prime}, A_{i 2}^{\prime \prime}, \cdots, A_{i n}^{\prime \prime}\right)$ holds for $i=1$, $2, \cdots, m$.

Ting-Yu CHEN is currently a Professor in the Graduate Institute of Business and Management and an Adjunct Professor in the Department of Industrial and Business Management at Chang Gung University in Taiwan. She received her BS degree in Transportation Engineering and Management, MS degree in Civil Engineering, and PhD degree in Traffic and Transportation from National Chiao Tung University in Taiwan. She has received a number of awards and grants. She is an Honorary Member of the Phi Tau Phi Scholastic Honor Society of Taiwan. Her current research interests include multiple criteria decision analysis, fuzzy decision-making methods and applications. 